\title{
MOMENTS OF THE ERROR TERM IN THE SATO-TATE LAW FOR ELLIPTIC CURVES
}

\author{
STEPHAN BAIER, NEHA PRABHU
}

\begin{abstract}
We derive new bounds for moments of the error in the Sato-Tate law over families of elliptic curves. Our estimates are stronger than those obtained in 44 and 5 for the first and second moment, but this comes at the cost of larger ranges of averaging. As applications, we deduce new almost-all results for the said errors and a conditional Central Limit Theorem on the distribution of these errors. Our method is different from those used in the above-mentioned papers and builds on recent work by the second-named author and K. Sinha [22] who derived a Central Limit Theorem on the distribution of the errors in the Sato-Tate law for families of cusp forms for the full modular group. In addition, identities by Birch and Melzak play a crucial rule in this paper. Birch's identities connect moments of coefficients of Hasse-Weil $L$-functions for elliptic curves with the Kronecker class number and further with traces of Hecke operators. Melzak's identity is combinatorial in nature.
\end{abstract}

\section{INTRODUCTION AND STATEMENT OF RESULTS}

For $(a, b) \in \mathbb{Z} \times \mathbb{Z}$ with $\Delta(a, b):=4 a^{3}+27 b^{2} \neq 0$ let $E(a, b)$ be the elliptic curve given in Weierstrass form by

$$
y^{2}=x^{3}+a x+b
$$

Consider the Hasse-Weil $L$-function

$$
L(E ; s):=\sum_{n=1}^{\infty} a_{E}(n) n^{-s}=\prod_{p \mid \mathcal{N}_{E}}\left(1-a_{E}(p) p^{-s}\right)^{-1} \prod_{p \nmid \mathcal{N}_{E}}\left(1-a_{E}(p) p^{-s}+p^{1-2 s}\right)^{-1} \quad(\Re(s)>1),
$$

where $\mathcal{N}_{E}$ is the conductor of $E$. By $\tilde{a}_{E}(n)$ we denote the normalized $n$-th coefficient, given by

$$
\tilde{a}_{E}(n):=\frac{a_{E}(n)}{\sqrt{n}} .
$$

It is due to Hasse that for $p$ prime, $\tilde{a}_{E}(p) \in[-2,2]$. The distribution of the sequence $\tilde{a}_{E}(p)$ in the interval $[-2,2]$ has been well investigated in the past few decades. For any interval $I \subseteq[-2,2]$ and elliptic curve $E$, let

$$
N_{I}(E, x):=\sharp\left\{x / 2<p \leq x: p \text { prime, } p \nmid \mathcal{N}_{E}, \tilde{a}_{E}(p) \in I\right\},
$$

where $\mathcal{N}_{E}$ is the conductor of $E$. Define $\tilde{\pi}(x)$ to be the number of primes between $x / 2$ and $x$. The Sato-Tate law for elliptic curves, conjectured independently by Sato and Tate around 1960 and recently proved by L. Clozel, M. Harris, N. Shepherd-Barron and R. Taylor (see [8, [12] and 24]), is equivalent to the following assertion about the distribution of the $\tilde{a}_{E}(p)$ 's in the interval $[-2,2]$.

Theorem 1.1. Let $E$ be an elliptic curve without complex multiplication over $\mathbb{Q}$ and $I$ be $a$ subinterval of $[-2,2]$. Then

$$
\lim _{x \rightarrow \infty} \frac{N_{I}(E, x)}{\tilde{\pi}(x)}=\int_{I} \frac{1}{\pi} \sqrt{1-\frac{t^{2}}{4}} d t .
$$


The Sato-Tate law has since been proved in full generality for Fourier coefficients of modular forms by T. Barnet-Lamb, D. Geraghty, M. Harris and R. Taylor (see [7]). We denote by $\mu(I)$ the Sato-Tate measure of any subinterval $I \subseteq[-2,2]$ given by the right hand side of (1).

In [4, the first-named author and L. Zhao established results which imply the following bounds for the first and second moments of the error $N_{I}(E, x)-\tilde{\pi}(x) \mu(I)$ in the Sato-Tate law over families of elliptic curves.

Theorem 1.2 (Zhao-Baier). Fix $\varepsilon>0$ and $c>0$. Let $I=[\alpha, \beta]$ be a subinterval of $(0,2]$. Suppose that $x^{\varepsilon-5 / 12} \leq(\beta-\alpha) / \beta \leq x^{-\varepsilon}$ and $\mu(I) \geq x^{\varepsilon-1 / 2}$. Then the following hold, where, by convention, the case when $\Delta(a, b)=4 a^{3}+27 b^{2}=0$ is excluded from the summations over a and $b$ below, and the $O$-constants depend only on $\varepsilon$ and $c$.

(i) If

$$
A, B \geq x^{1 / 2+\varepsilon} \quad \text { and } \quad A B \geq x^{1+\varepsilon} \mu(I)^{-1}
$$

then

(ii) If

$$
\frac{1}{4 A B} \sum_{|a| \leq A} \sum_{|b| \leq B}\left(N_{I}(E(a, b), x)-\tilde{\pi}(x) \mu(I)\right)=O_{\varepsilon, c}\left(\frac{\tilde{\pi}(x) \mu(I)}{(\log x)^{c}}\right) .
$$

then

$$
A, B \geq x^{1+\varepsilon} \quad \text { and } \quad x^{2+\varepsilon} \mu(I)^{-2} \leq A B \leq \exp \left(\exp \left(x^{1-\varepsilon}\right)\right)
$$

$$
\frac{1}{4 A B} \sum_{|a| \leq A} \sum_{|b| \leq B}\left(N_{I}(E(a, b), x)-\tilde{\pi}(x) \mu(I)\right)^{2}=O_{\varepsilon, c}\left(\frac{(\tilde{\pi}(x) \mu(I))^{2}}{(\log x)^{c}}\right) .
$$

Throughout the sequel, we want to keep the convention that the case $\Delta(a, b)=0$ is excluded from all summations over $a$ and $b$. We note that the summations in (3) and (4) include pairs $(a, b)$ such that $E(a, b)$ is a CM-curve, for which the Sato-Tate law is known not to hold by a result of M. Deuring [11]. However, the set of such pairs $(a, b)$ with $|a| \leq A$ and $|b| \leq B$ has cardinality $O\left(\min \left\{A^{1 / 2}, B^{1 / 3}\right\}\right)$ (see [3], for example), and therefore, the contribution of these pairs is negligible. $2])$.

The following almost-all result follows immediately from Theorem 1.2(ii) (see also 4, Corollary

Corollary 1.3. Fix $c, d>0$. Then, under the conditions of Theorem $1.2($ ii), we have

$$
\left|N_{I}(E(a, b), x)-\tilde{\pi}(x) \mu(I)\right| \ll \frac{\tilde{\pi}(x) \mu(I)}{(\log x)^{c}}
$$

for all $(a, b) \in \mathbb{Z}^{2}$ with $|a| \leq A$ and $|b| \leq B$, except for $O\left(A B(\log x)^{-d}\right)$ pairs $(a, b)$.

In 3], it was shown that (2) in Theorem 1.2(i) can be replaced by the condition

$$
A, B \geq x^{\varepsilon} \quad \text { and } \quad x^{1+\varepsilon} \mu(I)^{-1} \leq A B \leq x^{F},
$$

where $F$ is any positive constant. Even stronger bounds were obtained by Banks and Shparlinski [5] who obtained a power saving of $x^{\delta}$ over the trivial bound for the first moment if $I$ is fixed and

$$
A, B \geq x^{\varepsilon} \quad \text { and } \quad A B \geq x^{1+\varepsilon} .
$$

Here the size of $\delta$ depends on $\varepsilon$ and is smaller than $1 / 12$ (see equation (20) in [5]).

There are a number of related results in the literature (see, in particular, 99] and [21]). In this paper, we treat all moments, not only the first and second moments, and obtain new estimates. Our focus lies on strong savings over the trivial bounds rather than as small as possible families of curves (as weak as possible conditions on $A$ and $B$ ), which latter was the goal in the papers [4] and [5] as well as subsequent papers on this subject. Our savings for the first and second moments are indeed stronger than those obtained in [4] and [5]. In particular, for the first moment, we get, for fixed $I$, a saving of $x^{1 / 4}(\log x)^{c}$ unconditionally and $x^{1 / 2-\varepsilon}$ under MRH (a particular case of the Generalized Riemann Hypothesis, stated below) as compared to the power of logarithm saving in Theorem [1.2(i) and the above-mentioned saving of $x^{\delta}$ with $\delta<1 / 12$ obtained in [5]. The price of this improvement will be that our families of curves are larger, i.e., our conditions on $A$ and $B$ 
are stronger than those in Theorem 1.2, (6) and (7), but at a moderate level. More generally, we shall obtain power savings over the trivial bound for all moments.

To describe the results obtained, we require some notation. By $\sigma_{k}\left(T_{p}\right)$ we denote the trace and by $\tilde{\sigma}_{k}\left(T_{p}\right)$ the normalized trace of the Hecke operator $T_{p}$, acting on the space of cusp forms of weight $k$ for the full modular group, i.e.,

$$
\tilde{\sigma}_{k}\left(T_{p}\right)=\frac{\sigma_{k}\left(T_{p}\right)}{p^{\frac{k-1}{2}}} .
$$

In addition, we state a number of hypotheses below.

Modular Riemann Hypothesis - MRH: The Riemann Hypothesis holds for all $L$-functions associated to cusp forms $f \in S_{k}\left(\Gamma_{0}(1)\right)$ with $k \in \mathbb{N}$.

We note that

$$
\sum_{x / 2<p \leq x} \tilde{\sigma}_{k}\left(T_{p}\right)=O\left(k x^{1 / 2} \log k x\right)
$$

using the dimension formula for $S_{k}\left(\Gamma_{0}(1)\right)$ and the generalized prime number theorem (see Lemma 2.5 below). To prove asymptotic estimates rather than just bounds for the moments of the error in the Sato-Tate law and deduce a Central Limit Theorem on the distribution of this error, we will need the following plausible hypothesis which is slightly stronger on average over $k$ (namely, by a power of $\log x$ ).

Hypothesis 1. Let $c>0$ and $d_{2}>d_{1}>0$ be arbitrary but fixed. Then we have

$$
\sum_{k \leq K} \frac{1}{k} \cdot\left|\sum_{x / 2<p \leq x} \tilde{\sigma}_{k}\left(T_{p}\right)\right|=O_{c, d_{1}, d_{2}}\left(K x^{1 / 2}(\log x)^{-c}\right)
$$

as $x \rightarrow \infty$ if $d_{1} \log x \leq \log K \leq d_{2} \log x$.

We shall also use a second hypothesis which doensn't concern traces of Hecke operators.

Hypothesis 2. Let $c, d>0$ be arbitrary but fixed and suppose that $m \in \mathbb{N}$. Then we have

$$
\sum_{y<p \leq x} \tilde{a}_{E}\left(p^{m}\right)=O_{c, d}\left(m x(\log x)^{-c}\right)
$$

as $x \rightarrow \infty$ if $0 \leq y<x$ and $\log m \leq d \log \left(\mathcal{N}_{E} x\right)$.

The above Hypothesis 2 is true under Langland's conjectures (see [15]), which themselves imply the Sato-Tate law. To see this, one applies [13, Theorem 5.15], the generalized prime number theorem, to the symmetric power $L$-functions associated to $E$, which are automorphic and hence entire under the said conjectures, and uses the multiplicative properties of the coefficients $\tilde{a}_{E}(n)$ (see [18], for example).

Now we are ready to state our new moment bounds.

Theorem 1.4. Fix $c, \varepsilon>0$ and $t \in \mathbb{N}$. Set

$$
\eta(t):=\max \{t, 2(t-1)\}
$$

and

$$
\delta(t):= \begin{cases}1 & \text { if } t \text { is even } \\ 0 & \text { if } t \text { is odd }\end{cases}
$$


Suppose that $A, B \geq 1$ such that $A B \leq \exp \left(x^{1 / 2-\varepsilon}\right)$. Then we have

$$
\begin{aligned}
& \frac{1}{4 A B} \sum_{|a| \leq A} \sum_{|b| \leq B}\left(N_{I}(E(a, b), x)-\tilde{\pi}(x) \mu(I)\right)^{t} \\
= & \delta(t) \cdot \frac{t !}{2^{t / 2}(t / 2) !} \cdot\left(\mu(I)-\mu(I)^{2}\right)^{t / 2} \cdot \tilde{\pi}(x)^{t / 2}+ \\
& \begin{cases}O_{t, c, \varepsilon}\left(x^{3 t / 4}(\log x)^{-c}\right) & \text { unconditionally if } A, B \geq x^{\eta(t)+\varepsilon}, \\
O_{t, \varepsilon}\left(x^{t / 2}(\log x)^{t / 2}\right) & \text { under MRH if } A, B \geq x^{3 \eta(t) / 2+\varepsilon}, \\
O_{t, c, \varepsilon}\left(x^{t / 2}(\log x)^{-c}\right) & \text { under Hypotheses } 1,2 \text { if } A, B \geq x^{3 \eta(t) / 2+\varepsilon}, \\
O_{t, c, \varepsilon}\left(x^{t / 2}(\log x)^{-c}\right) & \text { under Hypothesis } 1 \text { if } A, B \geq x^{2 \eta(t)+\varepsilon} .\end{cases}
\end{aligned}
$$

The fourth bound above under Hypothesis 1 holds without assuming $A B \leq \exp \left(x^{1 / 2-\varepsilon}\right)$.

We point out that the main term on the right-hand side of (12) is dominated by the $O$-terms in the first two estimates, the unconditional one and the one under MRH, but not by the $O$-term in the third and fourth estimates under Hypotheses 1,2 if $I$ is not too short.

To achieve these results, we use a method which is different from those in [4] and [5], where the key point was the use of multiplicative characters to detect isomorphism classes of curves modulo primes. Our approach builds instead on the work [22] by the second-named author and K. Sinha about the distribution of the error in the Sato-Tate law for modular forms. Here the starting point is to detect the condition that $\tilde{a}_{E}(p) \in I$ by employing Theorem 1.8 below, which was established in 22 in the context of Fourier coefficients of cusp forms using Beurling-Selberg polynomials and the multiplicative properties of the coefficients in question. Then we use identities by Birch which connect moments of the coefficients $a_{E(a, b)}(p)$ with the Kronecker class number and further with traces of Hecke operators (see sections 6 and 7). This is followed by an application of Melzak's identity which is combinatorial in nature (see section 8). In this way, we connect two different kinds of families - families of elliptic curves and families of Hecke eigenforms for the full modular group.

Multiplicative characters are also applied in a similar fashion as in 44. (see section 5). This, however, is not essential for obtaining the savings in our estimates but only for lowering the sizes of our families of elliptic curves (see the remarks at the beginning of section 6 ). There may be some hope that these sizes can be reduced further by employing some ideas from [3] or [5].

As applications, we deduce new almost-all results which give support to a conditional estimate by K. Murty [19] and a conjecture by S. Akiyama and Y. Tanigawa [1] for individual curves. Moreover, we derive a Central Limit Theorem on the distribution of the error in the Sato-Tate law, conditional under Hypothesis 1. The said almost-all result is as follows and can be immediately deduced from the estimates for the second moment (case $k=2$ ) in Theorem 1.4 .

Corollary 1.5. Fix $c, \varepsilon>0$. Suppose that $A, B \geq 1$ such that $A B \leq \exp \left(x^{1 / 2-\varepsilon}\right)$ and $y>1$. Then for all pairs $(a, b) \in \mathbb{Z}^{2}$ with $|a| \leq A$ and $|b| \leq B$ with the exception of $O_{c, \varepsilon}\left(A B y^{-2}\right)$ pairs, we have

$$
\begin{aligned}
&\left|N_{I}(E(a, b), x)-\tilde{\pi}(x) \mu(I)\right| \\
& \leq \begin{cases}y x^{3 / 4}(\log x)^{-c} & \text { unconditionally if } A, B \geq x^{2+\varepsilon} \\
y x^{1 / 2}(\log x)^{1 / 2} & \text { under MRH if } A, B \geq x^{3+\varepsilon} \\
y\left(\left(\mu(I)-\mu(I)^{2}\right)^{1 / 2} \tilde{\pi}(x)^{1 / 2}+x^{1 / 2}(\log x)^{-c}\right) & \text { under Hypotheses } 1,2 \text { if } A, B \geq x^{3+\varepsilon} \\
y\left(\left(\mu(I)-\mu(I)^{2}\right)^{1 / 2} \tilde{\pi}(x)^{1 / 2}+x^{1 / 2}(\log x)^{-c}\right) & \text { under Hypothesis } 1 \text { if } A, B \geq x^{4+\varepsilon} .\end{cases}
\end{aligned}
$$

The fourth bound above under Hypothesis 1 holds without assuming $A B \leq \exp \left(x^{1 / 2-\varepsilon}\right)$.

K. Murty [19] proved that

$$
N_{I}(E, x)-\tilde{\pi}(x) \mu(I) \ll x^{3 / 4}\left(\log \mathcal{N}_{E} x\right)^{1 / 2}
$$

for every non-CM curve $E$, where $\mathcal{N}_{E}$ is the conductor of $E$, if all symmetric power $L$-functions associated to $E$ are automorphic and satisfy the Riemann Hypothesis. The first, unconditional, 
estimate in (13) gives support towards this conditional bound. It even shows that we have a slightly stronger bound for, in a sense, almost all curves $E$ (take, for example, $\left.y:=(\log x)^{c / 2}\right)$.

A conjecture by S. Akiyama and Y. Tanigawa [1] (see also the survey paper [16]) suggests that the bound

$$
\left|N_{I}(E, x)-\tilde{\pi}(x) \mu(I)\right| \ll_{E} x^{1 / 2+\varepsilon}
$$

should hold for all non-CM curves $E$, and there is numerical evidence in favor of it. The conditional estimates in (13) give support towards this conjecture (take, for example, $y:=x^{\varepsilon / 2}$ ). Moreover, the observation that the term $\left(\mu(I)-\mu(I)^{2}\right)^{1 / 2} \tilde{\pi}(x)^{1 / 2}$ in the third and fourth estimates cannot be removed gives rise to the conjecture that the exponent $1 / 2+\varepsilon$ in (14) is essentially optimal, which is supported by numerical data as well (see [16]).

As a second application of Theorem 1.4 the following Central Limit Theorem can be deduced from the last estimate for the moments in (12) under Hypothesis 1 by adapting the method of moments used in $[22$.

Theorem 1.6. Suppose that $A=A(x) \geq 1$ and $B=B(x) \geq 1$ satisfy $\frac{\log A}{\log x}, \frac{\log B}{\log x} \rightarrow \infty$ as $x \rightarrow \infty$. Assume that Hypothesis 1 holds. Then for any bounded continuous real function $h$ on $\mathbb{R}$, we have

$$
\lim _{x \rightarrow \infty} \frac{1}{4 A B} \sum_{|a| \leq A} \sum_{|b| \leq B} h\left(\frac{N_{I}(E(a, b), x)-\tilde{\pi}(x) \mu(I)}{\sqrt{\tilde{\pi}(x)\left(\mu(I)-\mu(I)^{2}\right)}}\right)=\frac{1}{\sqrt{2 \pi}} \int_{-\infty}^{\infty} h(t) e^{-t^{2} / 2} d t .
$$

This corresponds to the following unconditional Central Limit Theorem for the error in the Sato-Tate law for families of modular forms, established in [22. To understand the result, we first set up some notations. For any $N, k \in \mathbb{N}$ let $\mathcal{F}_{N, k}$ be an orthonormal basis of the subspace of all newforms in the space $S_{k}\left(\Gamma_{0}(N)\right)$ of cusp forms of weight $k$ with respect to $\Gamma_{0}(N)$.

(i) For any $f \in \mathcal{F}_{N, k}$, let

$$
f(z):=\sum_{n=1}^{\infty} a_{f}(n) q^{n} \quad \text { with } q=e^{2 \pi i z}
$$

be its Fourier expansion. By $\tilde{a}_{f}(n)$ we denote the normalized $n$-th coefficient, given by

$$
\tilde{a}_{f}(n):=\frac{a_{f}(n)}{n^{(k-1) / 2}} .
$$

(ii) For any interval $I \subseteq[-2,2]$ and $f \in \mathcal{F}_{N, k}$, let

$$
N_{I}(f, x):=\sharp\left\{p \leq x: p \text { prime, } p \nmid N, \tilde{a}_{f}(p) \in I\right\} .
$$

The said Central Limit Theorem established in 22 is as follows.

Theorem 1.7 (Prabhu-Sinha). Suppose that $k=k(x)$ satisfies $\frac{\log k}{\sqrt{x} \log x} \rightarrow \infty$ as $x \rightarrow \infty$. Then for any bounded continuous real function $h$ on $\mathbb{R}$, we have

$$
\lim _{x \rightarrow \infty} \frac{1}{\sharp \mathcal{F}_{1, k}} \sum_{f \in \mathcal{F}_{1, k}} h\left(\frac{N_{I}(f, x)-\pi(x) \mu(I)}{\sqrt{\pi(x)\left(\mu(I)-\mu(I)^{2}\right)}}\right)=\frac{1}{\sqrt{2 \pi}} \int_{-\infty}^{\infty} h(t) e^{-t^{2} / 2} d t .
$$

We now turn to the tools used to prove our main result, Theorem 1.4. A key result in [22, Section 4] that discusses the case of the first moment is the following approximation of the error in the Sato-Tate law for the case of Fourier coefficients of cusp forms. It will be essential in this work as well.

Theorem 1.8. Let $M \in \mathbb{N}$ and $I=[2 \cos \beta, 2 \cos \alpha] \subseteq[-2,2]$, where $0 \leq \alpha<\beta \leq \pi$. Then there exist real numbers $U_{I}^{-}(1), \ldots, U_{I}^{-}(M), U_{I}^{+}(1), \ldots, U_{I}^{+}(M)$ such that the following hold, where all $O$-constants below are absolute.

(i) We have

$$
U_{I}^{ \pm}(m)= \begin{cases}S_{I}^{ \pm}(m)-S_{I}^{ \pm}(m+2) & \text { if } m \leq M-2 \\ S_{I}^{ \pm}(m) & \text { if } m \in\{M-1, M\}\end{cases}
$$


where

(ii) Set

$$
S_{I}^{ \pm}(m):=\frac{\sin (2 \pi m \beta)-\sin (2 \pi m \alpha)}{m \pi}+O\left(\frac{1}{M}\right)
$$

$$
P_{I}^{ \pm}(E, x):=\sum_{1 \leq m \leq M} U_{I}^{ \pm}(m) \sum_{\substack{x / 2<p \leq x \\ p \nmid \mathcal{N}_{E}}} \tilde{a}_{E}\left(p^{m}\right) .
$$

Then

$$
P_{I}^{-}(E, x)+O\left(\frac{\tilde{\pi}(x)}{M}\right) \leq N_{I}(E, x)-\tilde{\pi}(x) \mu(I) \leq P_{I}^{+}(E, x)+O\left(\frac{\tilde{\pi}(x)}{M}\right) .
$$

An amazing and very useful fact, worked out in [20, is that the sum of the squares of coefficients $U_{I}^{ \pm}(m)$ above can be approximated using an expression depending on the Sato-Tate measure. This is the content of the following theorem.

Theorem 1.9. Let $M \geq 1$ and $U_{I}^{ \pm}(m)$ be defined as in Theorem 1.8 above. Then

$$
\sum_{1 \leq m \leq M} U_{I}^{ \pm}(m)^{2}=\mu(I)-\mu(I)^{2}+O\left(\frac{\log (2 M)}{M}\right)
$$

Now to prove Theorem 1.4, it shall suffice to establish the following two moment bounds.

Theorem 1.10. Fix $t \in \mathbb{N}$ and define $\delta(t)$ as in (11). Assume that $U(m)_{m \in \mathbb{N}}$ is a sequence of complex numbers such that

Let $M \geq 1$ and set

$$
U(m) \ll \frac{1}{m} \text { for all } m \in \mathbb{N} .
$$

$$
Z:=\sum_{1 \leq m \leq M} U(m)^{2}
$$

Fix $F, c, \varepsilon>0$. Suppose that $A, B \geq 1$ satisfy $A B \leq \exp \left(x^{1 / 2-\varepsilon}\right)$. Then we have

$$
\begin{aligned}
& \frac{1}{4 A B} \sum_{|a| \leq A} \sum_{|b| \leq B}\left(\sum_{1 \leq m \leq M} U(m) \sum_{\substack{x / 2<p \leq x \\
p \nmid a b \Delta(a, b)}} \tilde{a}_{E(a, b)}\left(p^{m}\right)\right)^{t} \\
= & \delta(t) \cdot \frac{t !}{2^{t / 2}(t / 2) !} \cdot Z^{t / 2}\left(\tilde{\pi}(x)^{t / 2}+O\left(\tilde{\pi}(x)^{t / 2-1}\right)\right)+ \\
& \begin{cases}O_{t, F, c, \varepsilon}\left(M^{t} x^{t / 2}(\log x)^{-c}\right) & \text { unconditionally if } x^{\varepsilon} \leq M \leq x^{F} \text { and } A, B \geq x^{t+\varepsilon} \\
O_{t, F, \varepsilon}\left(M^{t}(\log x)^{t}\right) & \text { under MRH if } \tilde{\pi}(x)^{1 / 2} \leq M \leq x^{F} \text { and } A, B \geq x^{3 t / 2+\varepsilon} \\
O_{t, F, c, \varepsilon}\left(M^{t}(\log x)^{-c}\right) & \text { under Hyp.1,2 if } \tilde{\pi}(x)^{1 / 2} \leq M \leq x^{F} \text { and } A, B \geq x^{3 t / 2+\varepsilon} .\end{cases}
\end{aligned}
$$

Theorem 1.11. Under the conditions of Theorem 1.10 with the condition $A B \leq \exp \left(x^{1 / 2-\varepsilon}\right)$ omitted, we have

$$
\begin{aligned}
& \frac{1}{4 A B} \sum_{|a| \leq A} \sum_{|b| \leq B}\left(\sum_{1 \leq m \leq M} U(m) \sum_{\substack{x / 2<p \leq x \\
p \nmid \Delta(a, b)}} \tilde{a}_{E(a, b)}\left(p^{m}\right)\right)^{t} \\
= & \delta(t) \cdot \frac{t !}{2^{t / 2}(t / 2) !} \cdot Z^{t / 2}\left(\tilde{\pi}(x)^{t / 2}+O\left(\tilde{\pi}(x)^{t / 2-1}\right)\right)+O_{t, F, c, \varepsilon}\left(M^{t}(\log x)^{-c}\right)
\end{aligned}
$$

if $\tilde{\pi}(x)^{1 / 2} \leq M \leq x^{F}$ and $A, B \geq x^{2 t+\varepsilon}$, provided that Hypothesis 1 holds.

We note that on the left-hand side of (20), the summation condition $p \nmid a b$, which is present in (19), is omitted. Avoiding this summation condition comes at the cost of a stronger condition on $A$ and $B$ in Theorem 1.11, as compared to Theorem 1.10, but on the other hand, we don't need to assume the truth of Hypothesis 2 , and the condition $A B \leq \exp \left(x^{1 / 2-\varepsilon}\right)$ is not needed either. 
Again, we point out that the main term on the right-hand side of (19) is dominated by the $O$ terms in the first two estimates, the unconditional one and the one under MRH, but not necessarily by the $O$-terms in the third estimate in (19) and in (20).

Our strategy of proof of Theorems 1.10 and 1.11 will be roughly as follows. First, we open up the $t$-th power. Then we reduce the products $\tilde{a}_{E(a, b)}\left(p_{1}^{m_{1}}\right) \cdots \tilde{a}_{E(a, b)}\left(p_{t}^{m_{t}}\right)$ arising in this way to linear combinations of terms of the form $\tilde{a}_{E(a, b)}(n)$, where the prime divisors of $n$ belong to the set $\left\{p_{1}, \ldots, p_{t}\right\}$. Now we pull in the sums over $a$ and $b$ and evaluate the averages of $\tilde{a}_{E(a, b)}(n)$ over $a$ and $b$. It turns out that they can be approximated using a multiplicative function $S(n)$ if $A$ and $B$ are large enough. This function $S(n)$ will be investigated further. Since it is multiplicative, it suffices to compute it at prime powers. To this end, we use identities by Birch and Melzak. Finally, we exploit the averaging over the primes $p_{1}, \ldots, p_{t}$ and the natural numbers $m_{1}, \ldots, m_{t}$. The main term will come from the contribution of $S(1)$.

In the following sections 2 to 9, we will provide the results that we need for the final proofs of Theorems 1.10 and 1.11, which will be carried out in sections 10 and 11, respectively.

Acknowledgements: We would like to thank Brundaban Sahu (NISER Bhubaneshwar) for having made SAGE computations which suggested the correctness of Lemma 8.1 providing a proof of Lemma 8.1 for the case $l=0$ and making us aware of the reference [17, which we used to prove the said lemma in full generality. The first-named author would like to thank the School of Physical Sciences at JNU Delhi for a great time. The second named author would like to thank IISER Pune for its resources and the National Board for Higher Mathematics for the PhD scholarship.

\section{Preliminaries}

2.1. Notations and basic facts. The following notations will be used throughout this paper.

Notations 1. (i) We reserve the symbol $p$ for primes greater or equal 5 and the symbol $E$ for elliptic curves over $\mathbb{Q}$, and we denote by $\mathcal{N}_{E}$ the conductor of $E$.

(ii) Throughout this paper, we assume that $x \geq 10$ and write

$$
\tilde{\pi}(x):=\pi(x)-\pi\left(\frac{x}{2}\right)=\sharp\{p \text { prime }: x / 2<p \leq x\} .
$$

(iii) Throughout this paper, we denote by I an arbitrary but fixed subinterval of $[-2,2]$ and

$$
\mu(I):=\int_{I} \frac{1}{\pi} \sqrt{1-\frac{t^{2}}{4}} d t
$$

We recall the following well-known facts on coeffients of Hasse-Weil $L$-functions associated to elliptic curves $E$ over $\mathbb{Q}$, which will be of key importance for our work (see [23, for example).

Theorem 2.1. (i) We have

$$
a_{E}(p)=\left\{\begin{array}{l}
p+1-\sharp E_{p} \text { if } E \text { has good reduction at } p \\
\in\{-1,0,1\} \text { otherwise, }
\end{array}\right.
$$

where $E_{p}$ is the curve over $\mathbb{F}_{p}$ obtained by reducing $E$ modulo $p$.

(ii) For every elliptic curve $E$ and every $n \in \mathbb{N}$, we have

$$
\left|\tilde{a}_{E}(n)\right| \leq d(n),
$$

where $d(n)$ is the number of divisors of $n$. In particular, if $p$ is a prime, then

$$
\tilde{a}_{E}(p) \in[-2,2] .
$$

(iii) The arithmetic functions $a_{E}: \mathbb{N} \rightarrow \mathbb{R}$ and $\tilde{a}_{E}: \mathbb{N} \rightarrow \mathbb{R}$ are multiplicative. 
(iv) For any prime $p$ at which $E$ has good reduction and any non-negative integers $i$ and $j$, we have

$$
\tilde{a}_{E}\left(p^{i}\right) \tilde{a}_{E}\left(p^{j}\right)=\sum_{l=0}^{\min (i, j)} \tilde{a}_{E}\left(p^{i+j-2 l}\right) .
$$

(v) For any prime $p$ at which $E$ has bad reduction and any non-negative integer $i$, we have

$$
\tilde{a}_{E}\left(p^{i}\right)=\tilde{a}_{E}(p)^{i} .
$$

We shall also use the following well-known dimension formula for the space $S_{k}\left(\Gamma_{0}(1)\right)$ of cusp forms for the full modular group in the course of this paper (see [14, for example).

Theorem 2.2. Let $k \in \mathbb{N}$. Then

$$
\operatorname{dim} S_{k}\left(\Gamma_{0}(1)\right)= \begin{cases}0 & \text { if } k \text { is odd } \\ 0 & \text { if } k=2 \\ \left\lfloor\frac{k}{12}\right\rfloor & \text { if } k \text { is even and } k \neq 2 \bmod 12 \\ \left\lfloor\frac{k}{12}\right\rfloor-1 & \text { if } k>2 \text { and } k \equiv 2 \bmod 12\end{cases}
$$

where for $z \in \mathbb{R},\lfloor z\rfloor$ denotes the largest integer not exceeding $z$.

2.2. Averages of traces of Hecke operators. In our paper, we shall establish a connection between families of elliptic curves and traces of Hecke operators. Recall

$$
\sigma_{k}\left(T_{p}\right)=\sum_{f \in \mathcal{F}_{1, k}} a_{f}(p) \text { and } \tilde{\sigma}_{k}\left(T_{p}\right)=\sum_{f \in \mathcal{F}_{1, k}} \tilde{a}_{f}(p) .
$$

We begin with collecting estimates for averages of these traces. Unconditionally, we have the following.

Lemma 2.3. Suppose that $k \in \mathbb{N}$. Then

$$
\sum_{x / 2<p \leq x} \tilde{\sigma}_{k}\left(T_{p}\right)=O\left(k x(\log k x)^{4} \exp (-C \sqrt{\log x})\right),
$$

where $C>0$ is a suitable constant.

Proof. Let $\mathcal{F}_{1, k}$ be the orthonormal basis of Hecke eigenforms of weight $k$ for the full modular group. Then, by [13, Theorem 5.13] (generalized prime number theorem), we have

$$
\sum_{x / 2<p \leq x} \tilde{a}_{f}(p)=O\left(x(\log k x)^{4} \exp (-C \sqrt{\log x})\right) \quad \text { if } f \in \mathcal{F}_{1, k}
$$

for some constant $C>0$, where the $O$-constant is absolute. This implies

$$
\sum_{x / 2<p \leq x} \tilde{\sigma}_{k}\left(T_{p}\right)=\sum_{f \in \mathcal{F}_{1, k}} \sum_{x / 2<p \leq x} \tilde{a}_{f}(p) \ll k x(\log k x)^{4} \exp (-C \sqrt{\log x})
$$

using Theorem 2.2

We shall also need the following bound with the same kind of saving by a factor of $\exp (-C \sqrt{\log x})$ for the average of the product of two traces of Hecke operators.

Lemma 2.4. Suppose that $k, l \in \mathbb{N}$. Then

$$
\sum_{x / 2<p \leq x} \tilde{\sigma}_{k}\left(T_{p}\right) \tilde{\sigma}_{l}\left(T_{p}\right)=O\left(k l x(\log k l x)^{4} \exp (-C \sqrt{\log x})\right),
$$

where $C>0$ is a suitable constant.

Proof. Let $\mathcal{F}_{1, k}$ and $\mathcal{F}_{1, l}$ be the orthonormal bases of Hecke eigenforms of weight $k$ and $l$ for the full modular group, respectively. Applying [13. Theorem 5.13] to the $L$-function associated to the Rankin-Selberg convolution $f \otimes g$ of $f \in \mathcal{F}_{1, k}$ and $g \in \mathcal{F}_{1, l}$, we have

$$
\sum_{x / 2<p \leq x} \tilde{a}_{f}(p) \tilde{a}_{g}(p)=O\left(x(\log k l x)^{4} \exp (-C \sqrt{\log x})\right)
$$


for some constant $C>0$, where the $O$-constant is absolute. This implies

$$
\sum_{x / 2<p \leq x} \tilde{\sigma}_{k}\left(T_{p}\right) \tilde{\sigma}_{l}\left(T_{p}\right)=\sum_{f \in \mathcal{F}_{1, k}} \sum_{g \in \mathcal{F}_{1, l}} \sum_{x / 2<p \leq x} \tilde{a}_{f}(p) \tilde{a}_{g}(p) \ll k l x(\log k l x)^{4} \exp (-C \sqrt{\log x})
$$

using Theorem 2.2

Under MRH, the following bound holds.

Lemma 2.5. Suppose that $k \in \mathbb{N}$. Then, under $\mathrm{MRH}$, we have

$$
\sum_{x / 2<p \leq x} \tilde{\sigma}_{k}\left(T_{p}\right)=O\left(k x^{1 / 2} \log k x\right),
$$

where the $O$-constant is absolute.

Proof. The proof follows the same lines as the proof of Lemma 2.3 above, but here we use 13 , Theorem 5.15] instead of [13, Theorem 5.13].

\section{Identities INVOLVING PRIME POWERS}

Theorem 2.1(iv) contains an identity which allows to write products of the form $\tilde{a}_{E}\left(p^{i}\right) \tilde{a}_{E}\left(p^{j}\right)$ as sums of terms of the form $\tilde{a}_{E}\left(p^{m}\right)$. The following Lemma provides a general result of this kind for products of the form $\tilde{a}_{E}\left(p^{m_{1}}\right) \cdots \tilde{a}_{E}\left(p^{m_{r}}\right)$ which was established in [22]. It will be used in the beginning of the proof of Theorem 1.10

Lemma 3.1. Assume that $m_{1}, \ldots, m_{r} \in \mathbb{N}$ and $E$ has good reduction at $p$. Let $s=m_{1}+\ldots+m_{r}$. Then

$$
\prod_{i=1}^{r} \tilde{a}_{E}\left(p^{m_{i}}\right)=\sum_{m=0}^{\infty} D\left(m_{1}, \ldots, m_{r} ; m\right) \tilde{a}_{E}\left(p^{m}\right)
$$

where $D\left(m_{1}, \ldots, m_{r} ; m\right)$ are nonnegative integers satisfying

$$
\begin{aligned}
D\left(m_{1}, \ldots, m_{r}, m\right) & =0 \quad \text { if } m>s, \\
D\left(m_{1}, \ldots, m_{r} ; m\right) & =O\left(s^{r-2}\right) \quad \text { if } r \geq 2 \text { and } 1 \leq m \leq s, \\
D\left(m_{1}, \ldots, m_{r} ; 0\right) & =O\left(s^{r-3}\right) \quad \text { if } r \geq 3, \\
D\left(m_{1}, m_{2} ; 0\right) & = \begin{cases}1 & \text { if } m_{1}=m_{2} \\
0 & \text { if } m_{1} \neq m_{2},\end{cases} \\
D\left(m_{1} ; m\right) & = \begin{cases}1 & \text { if } m_{1}=m \\
0 & \text { if } m_{1} \neq m .\end{cases}
\end{aligned}
$$

Further, it will be useful to express $\tilde{a}_{E}\left(p^{m}\right)$ as a polynomial in $\tilde{a}_{E}(p)$.

Lemma 3.2. Assume that $m \in \mathbb{N} \cup\{0\}$ and $E$ has good reduction at $p$. Define

$$
f_{m}(x):=\sum_{j=0}^{\lfloor m / 2\rfloor}(-1)^{j}\left(\begin{array}{c}
m-j \\
j
\end{array}\right) x^{m-2 j},
$$

where we set

$$
\left(\begin{array}{l}
0 \\
0
\end{array}\right)=1
$$

Then

$$
\tilde{a}_{E}\left(p^{m}\right)=f_{m}\left(\tilde{a}_{E}(p)\right) .
$$

Proof. We prove this lemma by induction over $m$. For $m=0,1$, (29) holds trivially. Assume that (29) holds for $m=k$. We show that (29) then holds for $m=k+2$.

By Theorem 2.1(iv), we have

$$
\tilde{a}_{E}\left(p^{k}\right) \tilde{a}_{E}\left(p^{2}\right)=\tilde{a}_{E}\left(p^{k+2}\right)+\tilde{a}_{E}\left(p^{k}\right)+\tilde{a}_{E}\left(p^{k-2}\right) .
$$


Hence,

$$
\tilde{a}_{E}\left(p^{k+2}\right)=\tilde{a}_{E}\left(p^{k}\right) \tilde{a}_{E}\left(p^{2}\right)-\tilde{a}_{E}\left(p^{k}\right)-\tilde{a}_{E}\left(p^{k-2}\right)=\tilde{a}_{E}\left(p^{k}\right)\left(\tilde{a}_{E}\left(p^{2}\right)-1\right)-\tilde{a}_{E}\left(p^{k-2}\right) .
$$

Further,

It follows that

$$
\tilde{a}_{E}(p)^{2}=\tilde{a}_{E}\left(p^{2}\right)+1
$$

By induction hypothesis, this implies that

$$
\tilde{a}_{E}\left(p^{k+2}\right)=\tilde{a}_{E}\left(p^{k}\right)\left(\tilde{a}_{E}(p)^{2}-2\right)-\tilde{a}_{E}\left(p^{k-2}\right) .
$$

$$
\begin{aligned}
\tilde{a}_{E}\left(p^{k+2}\right) & =\left(\tilde{a}_{E}(p)^{2}-2\right) \cdot \sum_{j=0}^{\lfloor k / 2\rfloor}(-1)^{j}\left(\begin{array}{c}
k-j \\
j
\end{array}\right) \tilde{a}_{E}(p)^{k-2 j}-\sum_{j=0}^{\lfloor k / 2-1\rfloor}(-1)^{j}\left(\begin{array}{c}
k-2-j \\
j
\end{array}\right) \tilde{a}_{E}(p)^{k-2-2 j} \\
& =\sum_{j=0}^{\lfloor k / 2+1\rfloor}(-1)^{j}\left(\left(\begin{array}{c}
k-j \\
j
\end{array}\right)+2\left(\begin{array}{c}
k-(j-1) \\
j-1
\end{array}\right)-\left(\begin{array}{c}
k-j \\
j-2
\end{array}\right)\right) \tilde{a}_{E}(p)^{k+2-2 j} \\
& =\sum_{j=0}^{\lfloor k / 2+1\rfloor}(-1)^{j}\left(\begin{array}{c}
k+2-j \\
j
\end{array}\right) \tilde{a}_{E}(p)^{k+2-2 j}
\end{aligned}
$$

which completes the proof.

We have the following bound which is consistent with Theorem 2.1(ii).

Lemma 3.3. Let $m \in \mathbb{N} \cup\{0\}$ and $-2 \leq x \leq 2$. Then

$$
\left|f_{m}(x)\right| \leq m+1 \text {. }
$$

Proof. The proof can be done directly, but an indirect argument based on the results we already stated seems the shortest. Fix an elliptic curve $E$. The coefficients $\tilde{a}_{E}(p)$ are known to satisfy the Sato-Tate law as $p$ varies over the primes of good reduction. In particular, the set

$$
\left\{\tilde{a}_{E}(p): p \text { prime, } p \nmid \mathcal{N}_{E}\right\}
$$

is dense in $[-2,2]$. The claim now follows from Theorem[2.1(ii), (29) and the continuity of $f_{m}$.

\section{Multiplicative structure of AVErages}

In this section, we exhibit that averages of $\tilde{a}_{E(a, b)}(n)$ over pairs $(a, b)$ in a box can be approximated using a multiplicative function in $n$. Our first result is the following approximation, which will later be refined.

Lemma 4.1. For all $A, B \geq 1$ and $n \in \mathbb{N}$,

$$
\sum_{\substack{|a| \leq A \\(a b \Delta(a, b), n)=1}} \tilde{a}_{E(a, b)}(n)=4 A B S(n)+O\left(d(n) s(n)^{2}\right)+O(d(n) s(n)(A+B)),
$$

where $s(n)$ is the largest squarefree number dividing $n$, and

$$
S(n):=\frac{1}{s(n)^{2}} \sum_{\substack{a=1 \\(a b \Delta(a, b), n)=1}}^{s(n)} \sum_{\substack{b=1 \\ s(n)}} \tilde{a}_{E(b)}(n) .
$$

Proof. First, we recall the inequality

$$
\left|\tilde{a}_{E(a, b)}(n)\right| \leq d(n)
$$

from Theorem 2.1(ii). It follows that

as well.

$$
|S(n)| \leq d(n)
$$

We observe that $\tilde{a}_{E(a, b)}(n)$ is doubly periodic in $a$ and $b$ with period $s(n)$ as is seen as follows. Since $\tilde{a}_{E(a, b)}\left(p^{m}\right)$ equals a polynomial in $\tilde{a}_{E(a, b)}(p)$ by Lemma 3.2. and $\tilde{a}_{E(a, b)}(p)$ is periodic in $a$ and $b$ with period $p$, respectively, it follows that $\tilde{a}_{E(a, b)}\left(p^{m}\right)$ is also periodic in $a$ and $b$ with period 
$p$, respectively. Since $\tilde{a}_{E(a, b)}(n)$ is multiplicative in $n$, we deduce that $\tilde{a}_{E(a, b)}(n)$ is periodic in $a$ and $b$ with period $s(n)$, respectively.

It follows that

$$
\begin{aligned}
& \sum_{\substack{|a| \leq A|b| \leq B \\
(a b \Delta(a, b), n)=1}} \tilde{a}_{E(a, b)}(n) \\
= & \sum_{-s(n)\left\lfloor\frac{A}{s(n)}\right\rfloor<a \leq s(n)\left\lfloor\frac{A}{s(n)}\right\rfloor-s(n)\left\lfloor\frac{B}{s(n)}\right\rfloor<b \leq s(n)\left\lfloor\frac{B}{s(n)}\right\rfloor} \sum_{\substack{(a b \Delta(a, b), n)=1 \\
=}} \tilde{a}_{E(a, b)}(n)+O(d(n) s(n)(s(n)+A+B)) \\
= & 4\left\lfloor\frac{A}{s(n)}\right\rfloor\left\lfloor\frac{B}{s(n)}\right\rfloor s(n)^{2} S(n)+O(d(n) s(n)(s(n)+A+B)) \\
= & 4 A B S(n)+O\left(d(n) s(n)^{2}\right)+O(d(n) s(n)(A+B)),
\end{aligned}
$$

which completes the proof.

Moreover, we prove the following.

Lemma 4.2. The function $S(n)$ defined in (32) is multiplicative.

Proof. Let $n_{1}, n_{2} \in \mathbb{N}$ such that $\left(n_{1}, n_{2}\right)=1$. Then, writing

$$
a=a_{1} s\left(n_{2}\right)+a_{2} s\left(n_{1}\right) \text { and } b=b_{1} s\left(n_{2}\right)+b_{2} s\left(n_{1}\right),
$$

we have

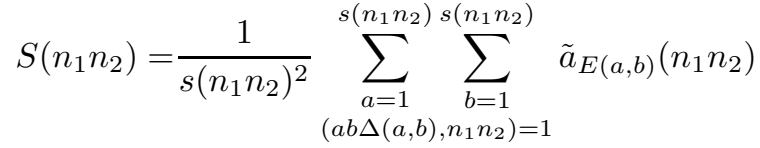

$$
\begin{aligned}
& =\frac{1}{s\left(n_{1}\right)^{2} s\left(n_{2}\right)^{2}} \sum_{a_{1}=1}^{s\left(n_{1}\right)} \sum_{a_{2}=1}^{s\left(n_{2}\right)} \sum_{b_{1}=1}^{s\left(n_{1}\right)} \sum_{b_{2}=1}^{s\left(n_{2}\right)} \tilde{a}_{E(a, b)}\left(n_{1}\right) \cdot \tilde{a}_{E(a, b)}\left(n_{2}\right) \\
& \left(a b \Delta(a, b), n_{1} n_{2}\right)=1 \\
& =\frac{1}{s\left(n_{1}\right)^{2} s\left(n_{2}\right)^{2}} \quad \sum_{a_{1}=1}^{s\left(n_{1}\right)} \sum_{a_{2}=1}^{s\left(n_{2}\right)} \sum_{b_{1}=1}^{s\left(n_{1}\right)} \sum_{b_{2}=1}^{s\left(n_{2}\right)} \quad \tilde{a}_{E\left(a_{1} s\left(n_{2}\right), b_{1} s\left(n_{2}\right)\right)}\left(n_{1}\right) \cdot \tilde{a}_{E\left(a_{2} s\left(n_{1}\right), b_{2} s\left(n_{1}\right)\right)}\left(n_{2}\right) \\
& \left(a_{1} b_{1} \Delta\left(a_{1}, b_{1}\right), n_{1}\right)=1=\left(a_{2} b_{2} \Delta\left(a_{2}, b_{2}\right), n_{2}\right) \\
& =\left(\frac{1}{s\left(n_{1}\right)^{2}} \sum_{\substack{a_{1}=1 \\
\left(a_{1} b_{1} \Delta\left(a_{1}, b_{1}\right), n_{1}\right)=1}}^{s\left(n_{1}\right)} \sum_{b_{1}=1}^{s\left(n_{1}\right)} \tilde{a}_{E\left(a_{1} s\left(n_{2}\right), b_{1} s\left(n_{2}\right)\right)}\left(n_{1}\right)\right) \times \\
& \left(\frac{1}{s\left(n_{2}\right)^{2}} \sum_{\substack{a_{2}=1 \\
\left(a_{2} b_{2} \Delta\left(a_{2}, b_{2}\right), n_{2}\right)=1}}^{s\left(n_{2}\right)} \sum_{b_{2}\left(n_{2}\right)} \tilde{a}_{E\left(a_{2} s\left(n_{1}\right), b_{2} s\left(n_{1}\right)\right)}\left(n_{2}\right)\right) \\
& =S\left(n_{1}\right) S\left(n_{2}\right) \text {, }
\end{aligned}
$$

which proves the claim.

\section{A Refined AVERAGe estimate}

Now we improve on the error term in Lemma 4.1. getting rid of the first $O$-term in (31) and saving a factor of $s(n)^{1 / 2-\varepsilon}$ in the second one. We mention that Lemma 4.1 would be already sufficient to prove a version of Theorem 1.4 , but with stronger conditions on $A$ and $B$. In particular, 
using Lemma 4.1 in our method, we can establish the first two estimates in (12) with the stronger conditions

$$
\begin{cases}A, B \geq x^{3 \eta(t) / 2+\varepsilon} & \text { unconditionally } \\ A, B \geq x^{2 \eta(t)}(\log x)^{-t / 2} & \text { under MRH }\end{cases}
$$

on $A$ and $B$. However, we are not content with these results and go for as weak as possible conditions on $A$ and $B$. To this end, we adapt the methods in [2] and [4, where refined asymptotic estimates for expressions of the form

$$
\frac{1}{4 A B} \sum_{r \in \mathcal{M}} \sum_{\substack{|a| \leq A \\ a_{E}(a, b)}} \sum_{\substack{|b| \leq B \\ a^{\prime}=r}} 1
$$

were established for $p$ prime and $\mathcal{M}$ a suitable set. Here we extend these considerations to arbitrary integers $n$ in place of primes $p$. We establish the following theorem. Since our proof follows closely the methods used in [2] and [4, we will cut some details.

Theorem 5.1. Let $\varepsilon>0$ be arbitrary but fixed. Then for all $A, B \geq 1$ and odd $n \in \mathbb{N}$, we have

$$
\sum_{\substack{|a| \leq A|b| \leq B \\(a b \Delta(a, b), n)=1}} \tilde{a}_{E(a, b)}(n)=4 A B S(n)+O_{\varepsilon, t}\left(d(n) s(n)^{1 / 2+\varepsilon}(A+B)\right),
$$

where $s(n)$ and $S(n)$ are defined as in Lemma 4.1 .

Proof. We first observe that the assertion is trivial if $n=1$. Therefore, we assume that $n>1$ throughout this proof and write the prime factorization of $n$ as $n=p_{1}^{m_{1}} \cdots p_{t}^{m_{t}}$.

5.1. Rewriting in terms of character sums. Using parts (ii) and (iii) of Theorem 2.1 and Lemma 3.2 into account, we write

$$
\begin{aligned}
& \sum_{\substack{|a| \leq A \\
(a b \Delta, b \mid \leq B \\
(a b \Delta(a, b), n)=1}} \tilde{a}_{E(a, b)}(n) \\
= & \sum_{-2 \sqrt{p_{1}} \leq r_{1} \leq 2 \sqrt{p_{1}}} \cdots \sum_{-2 \sqrt{p_{t} \leq r_{t} \leq 2 \sqrt{p_{t}}}} f_{m_{1}}\left(\frac{r_{1}}{\sqrt{p_{1}}}\right) \cdots f_{m_{t}}\left(\frac{r_{t}}{\sqrt{p_{t}}}\right) \sum_{\substack{|a| \leq A|b| \leq B \\
(a b \Delta(a, b), n)=1 \\
a_{E(a, b)}\left(p_{j}\right)=r_{j} \text { for } j \in\{1, \ldots, t\}}} 1 .
\end{aligned}
$$

For $p \nmid \Delta(a, b)$, let $E_{p}(a, b)$ be the elliptic curve over $\mathbb{F}_{p}$ obtained by reducing $E(a, b)$ modulo $p$. Similarly as in [2], we divide the inner-most double sum on the right-hand side of (36) into sums over isomorphism classes by writing

$$
\begin{aligned}
& \sum_{|a| \leq A} \sum_{|b| \leq B} \quad 1=\sum_{i_{1}=1}^{\mathcal{I}\left(p_{1} ; r_{1}\right)} \cdots \sum_{i_{t}=1}^{\mathcal{I}\left(p_{t} ; r_{t}\right)} \sum_{|a| \leq A|b| \leq B} \sum_{E_{I_{1}}} 1, \\
& (a b \Delta(a, b), n)=1 \quad E_{p_{1}}(a, b) \cong E_{p_{1}}\left(u\left(p_{1} ; r_{1}, i_{1}\right), v\left(p_{1} ; r_{1}, i_{1}\right)\right) \\
& a_{E(a, b)}\left(p_{j}\right)=r_{j} \text { for } j \in\{1, \ldots, t\} \quad E_{p_{t}}(a, b) \cong E_{p_{t}}\left(u\left(p_{t} ; r_{t}, i_{t}\right), v\left(p_{t} ; r_{t}, i_{t}\right)\right)
\end{aligned}
$$

where $\mathcal{I}\left(p_{j} ; r_{j}\right)$ are positive integers satisfying

$$
\mathcal{I}\left(p_{j} ; r_{j}\right) \leq H\left(r_{j}^{2}-4 p_{j}\right) \ll p_{j}^{1 / 2+\varepsilon},
$$

$H\left(r_{j}^{2}-4 p_{j}\right)$ being the Kronecker class number, $\left(u\left(p_{j} ; r_{j}, i_{j}\right), v\left(p_{j} ; r_{j}, i_{j}\right)\right)$ are suitable pairs of integers representing isomorphism classes and coprime to $p_{j}$, and $\cong$ indicates isomorphy of curves over $\mathbb{F}_{p}$. In [2], we used the fact that isomorphy of curves $E_{p}(a, b)$ and $E_{p}(u, v)$ over $\mathbb{F}_{p}$ can be described using congruence relations modulo $p$ involving the parameters $a, b, u, v$ (see Lemma 4 in 
[2]) and detected these relations using Dirichlet characters modulo $p$ (see equation (4.2) and the following equation in 2]). Applying this treatment to (37), we get

$$
\begin{aligned}
& \sum_{\substack{|a| \leq A \\
(a b \Delta(a, b), n)=1 \\
(a b)=B}} 1 \\
a_{E(a, b)}\left(p_{j}\right)=r_{j} \text { for } j \in\{1, \ldots, t\} & \sum_{i_{1}=1}^{\mathcal{I}\left(p_{1} ; r_{1}\right)} \cdots \sum_{i_{t}=1}^{\mathcal{I}\left(p_{t} ; r_{t}\right)} \sum_{|a| \leq A} \sum_{|b| \leq B} \prod_{j=1}^{t} F_{p_{j}}\left(a \cdot \overline{u\left(p_{j} ; r_{j}, i_{j}\right)}, b \cdot \overline{v\left(p_{j} ; r_{j}, i_{j}\right)}\right),
\end{aligned}
$$

where $\bar{z}$ is a multiplicative inverse of $z$ modulo $p_{j}$, i.e., $z \bar{z} \equiv 1 \bmod p_{j}$ if $p_{j} \nmid z$, and

$$
F_{p}(c, d):= \begin{cases}\frac{1}{4 \varphi(p)} \sum_{k=1}^{4}\left(\frac{c}{p}\right)_{4}^{k} \sum_{\bmod p} \chi\left(c^{3} \bar{d}^{2}\right) & \text { if } p \equiv 1 \bmod 4 \\ \frac{1}{4 \varphi(p)}\left(1+\left(\frac{c}{p}\right)_{2}\right)\left(1+\left(\frac{d}{p}\right)_{2}\right) \sum_{\chi \bmod p} \chi\left(c^{3} \bar{d}^{2}\right) & \text { if } p \equiv 3 \bmod 4,\end{cases}
$$

$(\cdot / p)_{w}$ being the $w$-th power residue symbol.

5.2. Division into main and error terms. At this point, we follow the method in 2], where we treated only the case $t=1$ of one prime. We will therefore be brief at some places. Similarly as in 2] and [4, we only deal with the case when $p_{1}, \ldots, p_{t}$ are all congruent 1 modulo 4 . The general case can be handled similarly, but we need to divide into more character sums. If we are in the said case $p_{1}, \ldots, p_{t} \equiv 1 \bmod 4$, then using the Chinese Remainder Theorem, we can simplify (39) into

$$
\begin{aligned}
& \sum_{\substack{|a| \leq A|b| \leq B \\
a b \Delta(a, b), n)=1}} \quad 1=\frac{1}{4^{\omega(n)} \varphi(s(n))} \sum_{i=1}^{\mathcal{I}\left(n ; r_{1}, \ldots, r_{t}\right)} \sum_{\substack{\bmod s(n) \\
\operatorname{ord}(\tilde{\chi}) \mid 4}} \sum_{\chi \bmod s(n)} \sum_{|a| \leq A|b| \leq B} \\
& \tilde{\chi}\left(a \overline{u_{i}\left(n ; r_{1}, \ldots, r_{t}\right)}\right) \chi\left(a^{3} \bar{b}^{2} \overline{u_{i}\left(n ; r_{1}, \ldots, r_{t}\right)^{3}} v_{i}\left(n ; r_{1}, \ldots, r_{t}\right)^{2}\right),
\end{aligned}
$$

where

$$
\mathcal{I}\left(n ; r_{1}, \ldots, r_{t}\right)=\prod_{j=1}^{t} \mathcal{I}\left(p_{j} ; r_{j}\right)
$$

and $\left(u_{i}\left(n ; r_{1}, \ldots, r_{t}\right), v_{i}\left(n ; r_{1}, \ldots, r_{t}\right)\right)$ are suitable pairs of integers. Similarly as in 2] and [4, we divide the right-hand side of (40) into a main and error term, where the main term is the contribution of characters $\tilde{\chi}$ and $\chi$ such that $\tilde{\chi} \chi^{3}=\chi_{0}=\chi^{2}, \chi_{0}$ being the principal character modulo $p$, and the error term is the remaining contribution. Using (36) and (40), it follows that

$$
\sum_{\substack{|a| \leq A|b| \leq B \\(a b \Delta(a, b), n)=1}} \tilde{a}_{E(a, b)}(n)=M(n ; A, B)+E(n ; A, B),
$$

where

$$
\begin{gathered}
M(n ; A, B):=\frac{1}{4^{\omega(n)} \varphi(s(n))}\left(\sum_{\substack{\tilde{\chi} \bmod s(n) \chi \bmod s(n) \\
\operatorname{ord}(\tilde{\chi}) \mid 4 \\
\tilde{\chi} \chi^{3}=\chi_{0}=\chi^{2}}} 1\right) \cdot\left(\sum_{\substack{|a| \leq A|b| \leq B \\
(a b, n)=1}} 1\right) \times \\
\left(\sum_{-2 \sqrt{p_{1} \leq r_{1} \leq 2 \sqrt{p_{1}}}} \cdots \sum_{-2 \sqrt{p_{t} \leq r_{t} \leq 2 \sqrt{p_{t}}}} f_{m_{1}}\left(\frac{r_{1}}{\sqrt{p_{1}}}\right) \cdots f_{m_{t}}\left(\frac{r_{t}}{\sqrt{p_{t}}}\right) \sharp \mathcal{I}\left(n ; r_{1}, \ldots, r_{t}\right)\right)
\end{gathered}
$$


and

$$
\begin{aligned}
& E(n ; A, B):=\frac{1}{4^{\omega(n)} \varphi(s(n))} \sum_{\substack{\tilde{\chi} \bmod s(n) \chi \bmod s(n) \\
\text { ord }(\tilde{\chi}) \mid 4 \\
\tilde{\chi} \chi^{3} \neq \chi_{0} \text { or } \chi^{2} \neq \chi_{0}}} \sum_{\substack{\sum_{-2 \sqrt{p_{1}} \leq r_{1} \leq 2 \sqrt{p_{1}}} \\
\sum_{i=1}^{\mathcal{I}\left(n ; r_{1}, \ldots, r_{t}\right)}}} \sum_{-2 \sqrt{p_{t} \leq r_{t} \leq 2 \sqrt{p_{t}}}} f_{m_{1}}\left(\frac{r_{1}}{\sqrt{p_{1}}}\right) \cdots f_{m_{t}}\left(\frac{r_{t}}{\sqrt{p_{t}}}\right) \times \\
& \left(\sum_{|a| \leq A} \tilde{\chi} \chi^{3}(a)\right)\left(\sum_{|b| \leq B} \bar{\chi}^{2}(b)\right) .
\end{aligned}
$$

5.3. Treatment of the main term. We first relate $M(n ; A, B)$ to the main term on the righthand side (31). This is based on two observations. Firstly,

$$
\begin{aligned}
M(n ; A, B) & =\left(\sum_{\substack{|a| \leq A|b| \leq B \\
(a b, n)=1}} 1\right)\left(\sum_{\substack{|a| \leq s(n) \\
(a b, n)=1}} \sum_{\substack{|b| \leq(n) \\
(a b)}} 1\right)^{-1} M(n ; s(n), s(n)) \\
& =\frac{1}{4 \varphi(s(n))^{2}}\left(\sum_{\substack{|a| \leq A|b| \leq B \\
(a b, n)=1}} 1\right) M(n ; s(n), s(n)),
\end{aligned}
$$

and secondly,

$$
E(n ; s(n), s(n))=0
$$

by the orthogonality relations for Dirichlet characters. From the definition of $S(n)$ in (32) and the equations (42) and (46) above, it follows that

$$
4 s(n)^{2} S(n)=\sum_{\substack{|a| \leq s(n) \\(a b \Delta(a, b), n)=1}} \sum_{\substack{\mid b(n) \\ E(a, b)}}(n)=M(n ; s(n), s(n)) .
$$

From (45) and (47), we conclude that

$$
M(n ; A, B)=\left(\frac{s(n)}{\varphi(s(n))}\right)^{2}\left(\sum_{\substack{|a| \leq A \\(a b, n)=1}} \sum_{|b| \leq B} 1\right) S(n)=\left(\frac{n}{\varphi(n)}\right)^{2}\left(\sum_{\substack{|a| \leq A|b| \leq B \\(a b, n)=1}} 1\right) S(n) .
$$

Detecting the summation condition $(a b, n)=1$ using the Möbius function, it is easily seen that

$$
\sum_{\substack{|a| \leq A|b| \leq B \\(a b, n)=1}} 1=4 A B\left(\frac{\varphi(n)}{n}\right)^{2}+O\left(2^{t}(A+B)+4^{t}\right) .
$$

Putting (48) and (49) together, and using (33), we obtain

$$
M(n ; A, B)=4 A B S(n)+O_{t}\left(d(n)\left(\frac{n}{\varphi(n)}\right)^{2}(A+B)\right) .
$$




\subsection{Treatment of the error term. Set}

$$
\mathcal{I}_{n}:=\sum_{-2 \sqrt{p_{1}} \leq r_{1} \leq 2 \sqrt{p_{1}}} \cdots \sum_{-2 \sqrt{p_{t}} \leq r_{t} \leq 2 \sqrt{p_{t}}} \mathcal{I}\left(n ; r_{1}, \ldots, r_{t}\right) .
$$

For the error term $E(n ; A, B)$, defined in (44), we employ the same method as in [4] based on the Polya-Vinogradov inequality and bounds for the second and fourth moments of character sums, getting

$$
E(n ; A, B)=O\left(s(n)^{\varepsilon} d(n)\left(\mathcal{I}_{n}(A+B) s(n)^{-1 / 2}+\left(\mathcal{I}_{n} A B\right)^{1 / 2}\right)\right),
$$

where we also use the bound

$$
\left|f_{m_{1}}\left(\frac{r_{1}}{\sqrt{p_{1}}}\right) \cdots f_{m_{t}}\left(\frac{r_{t}}{\sqrt{p_{t}}}\right)\right| \leq\left(m_{1}+1\right) \cdots\left(m_{t}+1\right)=d(n)
$$

following from Lemma 3.3. Combining (38), (41) and (51), we have

$$
\mathcal{I}_{n}=O_{\varepsilon}\left(s(n)^{1+\varepsilon}\right),
$$

and hence, taking into account that $(A B)^{1 / 2} \ll A+B$, we deduce from (52) that

$$
E(n ; A, B)=O_{\varepsilon, t}\left(d(n) s(n)^{1 / 2+\varepsilon}(A+B)\right) .
$$

Now the claimed asymptotic estimate (35) follows from (42), (501), (53) and

$$
\frac{n}{\varphi(n)} \ll_{\varepsilon, t} s(n)^{\varepsilon} .
$$

This completes the proof.

\section{Relation to the Kronecker Class number}

Next, we evaluate the multiplicative function $S(n)$, exhibited in the last two sections, at prime powers. Recalling its definition in (32) and our general condition $p \geq 5$, we write

$$
S\left(p^{m}\right)=S_{0}\left(p^{m}\right)-S_{1}\left(p^{m}\right)-S_{2}\left(p^{m}\right),
$$

where

$$
\begin{gathered}
S_{0}\left(p^{m}\right):=\frac{1}{p^{2}} \sum_{\substack{a=1 \\
p \nmid \Delta(a, b)}}^{p} \sum_{b=1}^{p} \tilde{a}_{E(a, b)}\left(p^{m}\right), \\
S_{1}\left(p^{m}\right):=\frac{1}{p^{2}} \sum_{a=1}^{p-1} \tilde{a}_{E(a, 0)}\left(p^{m}\right) \text { and } S_{2}\left(p^{m}\right):=\frac{1}{p^{2}} \sum_{b=1}^{p-1} \tilde{a}_{E(0, b)}\left(p^{m}\right) .
\end{gathered}
$$

We first deal with $S_{0}\left(p^{m}\right)$ and handle $S_{1,2}\left(p^{m}\right)$ later.

Using Lemma 3.2, we obtain

$$
\begin{aligned}
S_{0}\left(p^{m}\right) & =\frac{1}{p^{2}} \sum_{j=0}^{[m / 2]}(-1)^{j}\left(\begin{array}{c}
m-j \\
j
\end{array}\right) \sum_{\substack{a=1 \\
p \nmid \Delta(a, b)}}^{p} \sum_{\substack{b=1 \\
p(a, b)}}^{p}(p)^{m-2 j} \\
& =\frac{1}{p^{2}} \sum_{j=0}^{[m / 2]}(-1)^{j}\left(\begin{array}{c}
m-j \\
j
\end{array}\right) \cdot \frac{1}{p^{m / 2-j}} \sum_{\substack{a=1 \\
p \nmid \Delta(a, b)}}^{p} \sum_{b=1}^{p} a_{E(a, b)}(p)^{m-2 j} .
\end{aligned}
$$

Considering the arguments in [6], the following expression for the double sum over $a$ and $b$ in the last line holds. 
Lemma 6.1. For any prime $p \geq 5$ and positive integer $g$,

$$
\sum_{\substack{a=1 \\ p \nmid \Delta(a, b)}}^{p} \sum_{\substack{b=1 \\ \text { p }}}^{p} a_{E(a, b)}(p)^{g}=\frac{p-1}{2} \sum_{|r| \leq 2 \sqrt{p}} r^{g} H\left(r^{2}-4 p\right),
$$

where $H\left(r^{2}-4 p\right)$ is the Kronecker class number, and the O-constant is absolute.

If $g$ is odd, this gives

$$
\sum_{\substack{a=1 \\ p \nmid \Delta(a, b)}}^{p} \sum_{E(a, b)}^{p}(p)^{g}=0
$$

which implies

$$
S_{0}\left(p^{m}\right)=0 \quad \text { if } m \text { is odd. }
$$

If $m=2 k$ is even, then using (57) and (58), we obtain

$$
\begin{aligned}
S_{0}\left(p^{2 k}\right) & :=\frac{1-1 / p}{p^{k+1}} \sum_{j=0}^{k}(-1)^{j}\left(\begin{array}{c}
2 k-j \\
j
\end{array}\right) p^{j} \cdot \frac{1}{2} \sum_{|r| \leq 2 \sqrt{p}} r^{2(k-j)} H\left(r^{2}-4 p\right) \\
& =\frac{1-1 / p}{p^{k+1}} \sum_{j=0}^{k}(-1)^{k-j}\left(\begin{array}{c}
k+j \\
k-j
\end{array}\right) p^{k-j} \cdot \frac{1}{2} \sum_{|r| \leq 2 \sqrt{p}} r^{2 j} H\left(r^{2}-4 p\right) .
\end{aligned}
$$

\section{An identity By BiRCH}

Now we use the following identity due to Birch [6].

Lemma 7.1. For every prime $p \geq 5$ and positive integer $j$,

$$
\begin{aligned}
\frac{1}{2} \sum_{|r| \leq 2 \sqrt{p}} r^{2 j} H\left(r^{2}-4 p\right)= & \frac{(2 j) !}{j !(j+1) !} \cdot p^{j+1} \\
& -\sum_{l=1}^{j}(2 l+1) \cdot \frac{(2 j) !}{(j-l) !(j+l+1) !} \cdot p^{j-l}\left(\sigma_{2(l+1)}\left(T_{p}\right)+1\right),
\end{aligned}
$$

where $\sigma_{2(l+1)}\left(T_{p}\right)$ is the trace of the Hecke operator $T_{p}$ acting on the space of cusp forms of weight $2(l+1)$ for the full modular group.

Plugging (61) into the last line of (60) and re-arranging summations gives

$$
\begin{aligned}
S_{0}\left(p^{2 k}\right)= & \frac{1-1 / p}{p^{k+1}} \sum_{j=0}^{k}(-1)^{k-j}\left(\begin{array}{l}
k+j \\
k-j
\end{array}\right) p^{k-j} \times \\
& \left(\frac{(2 j) !}{j !(j+1) !} \cdot p^{j+1}-\sum_{l=1}^{j}(2 l+1) \cdot \frac{(2 j) !}{(j-l) !(j+l+1) !} \cdot p^{j-l}\left(\sigma_{2(l+1)}\left(T_{p}\right)+1\right)\right) \\
= & \left(1-\frac{1}{p}\right)\left(A_{0, k}-\sum_{l=1}^{k} A_{l, k} p^{-(l+1)}\left(\sigma_{2(l+1)}\left(T_{p}\right)+1\right)\right),
\end{aligned}
$$

where

$$
A_{l, k}:=(2 l+1) \sum_{j=l}^{k}(-1)^{k-j}\left(\begin{array}{c}
k+j \\
k-j
\end{array}\right) \cdot \frac{(2 j) !}{(j-l) !(j+l+1) !} .
$$




\section{An identity by MeLzak}

We claim the following.

Lemma 8.1. For any nonnegative integers $k, l$ with $0 \leq l \leq k$, we have

$$
A_{l, k}= \begin{cases}0 & \text { if } l<k \\ 1 & \text { if } l=k\end{cases}
$$

Proof. It is clear that this holds if $l=k$. For the case when $l<k$, we use Melzak's identity (see [17]) which states that

$$
f(x+y)=x\left(\begin{array}{c}
x+n \\
n
\end{array}\right) \sum_{a=0}^{n}(-1)^{a}\left(\begin{array}{l}
n \\
a
\end{array}\right) \cdot \frac{f(y-a)}{x+a}
$$

for all polynomials of degree up to $n$ and $x \notin\{0,-1, \ldots,-n\}$. Rearranging factors, and making a change of variables $n=k+l$ and $a=j+l$, it is easily seen that

$$
\begin{aligned}
A_{l, k} & =(2 l+1) \sum_{j=l}^{k}(-1)^{k-j}\left(\begin{array}{c}
k+j \\
k+l
\end{array}\right)\left(\begin{array}{c}
k+l \\
j+l
\end{array}\right) \cdot \frac{1}{j+l+1} \\
& =(2 l+1) \sum_{a=2 l}^{n}(-1)^{n-a}\left(\begin{array}{c}
n+a-2 l \\
n
\end{array}\right)\left(\begin{array}{l}
n \\
a
\end{array}\right) \cdot \frac{1}{a+1} .
\end{aligned}
$$

Now we set $x=1, y=0$ and

$$
f(z):=\frac{(n-z-2 l)(n-1-z-2 l) \cdots(1-z-2 l)}{n !} .
$$

Then it follows that

$$
A_{l, k}=(2 l+1)(-1)^{n} \sum_{a=0}^{n}(-1)^{a}\left(\begin{array}{l}
n \\
a
\end{array}\right) \cdot \frac{f(y-a)}{x+a},
$$

and (64) therefore gives

$$
\begin{aligned}
A_{l, k} & =(2 l+1)(-1)^{n} \cdot \frac{f(x+y)}{x\left(\begin{array}{c}
x+n \\
n
\end{array}\right)}=(2 l+1)(-1)^{n} \cdot \frac{f(1)}{n+1} \\
& =(2 l+1)(-1)^{n} \cdot \frac{(n-1-2 l)(n-2-2 l) \cdots(-2 l)}{(n+1) !}=0
\end{aligned}
$$

since $n-1-2 l=k-l-1 \geq 0$. This completes the proof.

Now, combining (59), (62) and Lemma 8.1, we obtain the following.

Lemma 8.2. For any prime $p \geq 5$ and $m \in \mathbb{N}$, we have

$$
S_{0}\left(p^{m}\right)=\left(1-p^{-1}\right) p^{-(m / 2+1)} \sigma_{m+2}\left(T_{p}\right),
$$

where $\sigma_{m+2}\left(T_{p}\right)=0$ if $m$ is odd.

\section{Averages over Prime powers}

Now we want to bound averages of $S\left(p^{m}\right)$ and, more generally, products of the form $S\left(p^{m_{1}}\right) \cdots S\left(p^{m_{r}}\right)$ over primes. To this end, we first handle the functions $S_{1,2}\left(p^{m}\right)$, defined in (56), which is easy.

Lemma 9.1. Let $c, d>0$ be arbitrary but fixed and $m \in \mathbb{N}$. Then the following hold.

(i) We have

(ii) Under Hypothesis 2, we have

$$
S_{1,2}\left(p^{m}\right)=O\left(\frac{m}{p}\right)
$$

$$
\sum_{x / 2<p \leq x} S_{1,2}\left(p^{m}\right)=O_{c, d}\left(\frac{m}{(\log x)^{c}}\right)
$$

if $\log m \leq d \log x$. 
Proof. Part (i) is a direct consequence of Theorem 2.1(ii), and part (ii) follows from Hypothesis 2 and partial summation after re-arranging summations in the form

and

$$
\sum_{x / 2<p \leq x} S_{1}\left(p^{m}\right)=\sum_{a=1}^{x-1} \sum_{\max \{x / 2, a\}<p \leq x} \frac{\tilde{a}_{E(a, 0)}\left(p^{m}\right)}{p^{2}}
$$

$$
\sum_{x / 2<p \leq x} S_{2}\left(p^{m}\right)=\sum_{b=1}^{x-1} \sum_{\max \{x / 2, b\}<p \leq x} \frac{\tilde{a}_{E(0, b)}\left(p^{m}\right)}{p^{2}} .
$$

From Lemmas 8.2 and 9.1 , we deduce the following average results.

Lemma 9.2. Let $c, d>0$ and $d_{2}>d_{1}>0$ be arbitrary but fixed. Then the following hold.

(i) Let $m \in \mathbb{N}$. Then, unconditionally, we have

$$
\sum_{x / 2<p \leq x} S\left(p^{m}\right)=O_{c}\left(m x^{1 / 2}(\log x)^{-c}\right) .
$$

(ii) Let $m \in \mathbb{N}$. Assume that $\log m \leq d \log x$. Then, under $\mathrm{MRH}$, we have

$$
\sum_{x / 2<p \leq x} S\left(p^{m}\right)=O_{d}(m \log x) .
$$

(iii) Assume that $d_{1} \log x \leq \log M \leq d_{2} \log x$. Then, under Hypotheses 1 and 2 , we have

$$
\sum_{1 \leq m \leq M} \frac{1}{m} \cdot\left|\sum_{x / 2<p \leq x} S\left(p^{m}\right)\right|=O_{c, d_{1}, d_{2}}\left(M(\log x)^{-c}\right) .
$$

(iv) Let $m_{1}, m_{2} \in \mathbb{N}$. Assume that $\log m_{1,2} \leq d \log x$. Then

$$
\sum_{x / 2<p \leq x} S\left(p^{m_{1}}\right) S\left(p^{m_{2}}\right)=O_{c, d}\left(m_{1} m_{2}(\log x)^{-c}\right) .
$$

(v) Let $r \geq 2$ and $m_{1}, \ldots, m_{r} \in \mathbb{N}$. Then

$$
\sum_{x / 2<p \leq x} S\left(p^{m_{1}}\right) \cdots S\left(p^{m_{r}}\right)=O_{r}\left(\frac{m_{1} \cdots m_{r}}{x^{r / 2-1} \log x}\right) .
$$

Proof. The claimed bounds in (i)-(iv) follow from (54), Lemma 8.2. Lemma 9.1 and Lemmas 2.3. 2.4, 2.5] as well as Hypotheses 1,2, respectively, using partial summation. To prove part (v), we use (23), Theorem 2.2, (54), Lemmas 8.2 and 9.1(i) and the Deligne bound (see [10])

$$
\left|a_{f}(p)\right| \leq 2 p^{(m+1) / 2} \quad \text { if } f \in \mathcal{F}_{1, m+2}
$$

to obtain

$$
S\left(p^{m}\right) \ll \frac{\sharp \mathcal{F}_{1, m+2}}{\sqrt{p}}+\frac{m}{p} \ll \frac{m}{p^{1 / 2}}
$$

for all primes $p \geq 5$ and $m \in \mathbb{N}$, from which the claimed bound (66) follows using

$$
\tilde{\pi}(x) \sim \frac{x}{2 \log x}
$$

by the prime number theorem.

We remark that the power savings obtained in Theorem 1.4 depend on the fact that we have a nontrivial estimate for $S_{0}\left(p^{m}\right)$ above, with a saving by a factor of $\sqrt{p}$ over the trivial bound $S_{0}\left(p^{m}\right)=O(m)$.

In addition, we record the following bound for the average of $S_{0}\left(p^{m}\right)$ which can be proved in the same way as Lemma 9.2 (iii) above, where Hypothesis 2 is not required. 
Lemma 9.3. Let $c>0$ and $d_{2}>d_{1}>0$ be arbitrary but fixed. Assume that $d_{1} \log x \leq \log M \leq$ $d_{2} \log x$. Then, under Hypotheses 1 , we have

$$
\sum_{1 \leq m \leq M} \frac{1}{m} \cdot\left|\sum_{x / 2<p \leq x} S_{0}\left(p^{m}\right)\right|=O_{c, d_{1}, d_{2}}\left(M(\log x)^{-c}\right) .
$$

\section{Proof of Theorem 1.10}

Now we are ready to prove Theorem 1.10, a key result in this paper.

10.1. Opening up the $t$-th power. Throughout the sequel, we write

$$
X_{t}:=\frac{1}{4 A B} \sum_{|a| \leq A} \sum_{|b| \leq B}\left(\sum_{1 \leq m \leq M} U(m) \sum_{\substack{x / 2<p \leq x \\ p \nmid a b \Delta(a, b)}} \tilde{a}_{E(a, b)}\left(p^{m}\right)\right)^{t} .
$$

Opening the $t$-power, we get

$$
\begin{aligned}
& X_{t}=\frac{1}{4 A B} \sum_{|a| \leq A} \sum_{|b| \leq B} \sum_{1 \leq m_{1}, \ldots, m_{t} \leq M} U\left(m_{1}\right) \cdots U\left(m_{t}\right) \times \\
& \sum_{\substack{x / 2<p_{1}, \ldots, p_{t} \leq x \\
\left(a b \Delta(a, b), p_{1} \cdots p_{t}\right)=1}} \tilde{a}_{E(a, b)}\left(p_{1}^{m_{1}}\right) \cdots \tilde{a}_{E(a, b)}\left(p_{t}^{m_{t}}\right) .
\end{aligned}
$$

Further, we write

$$
\begin{aligned}
& \sum_{\substack{x / 2<p_{1}, \ldots, p_{t} \leq x \\
\left(a b \Delta(a, b), p_{1} \cdots p_{t}\right)=1}} \tilde{a}_{E(a, b)}\left(p_{1}^{m_{1}}\right) \cdots \tilde{a}_{E(a, b)}\left(p_{t}^{m_{t}}\right) \\
= & \sum_{u=1}^{t} \sum_{\substack {\{1, \ldots, t\}=\mathcal{S}_{1} \dot{\cup} \ldots \cup \mathcal{S}_{u} \\
\begin{subarray}{c}{x / 2<p_{1}, \ldots, p_{u} \leq x \\
\left(a b \Delta(a, b), p_{1} \cdots p_{u}\right)=1 \\
p_{r} \neq p_{s} \text { if } 1 \leq r<s \leq u{ \{ 1 , \ldots , t \} = \mathcal { S } _ { 1 } \dot { \cup } \ldots \cup \mathcal { S } _ { u } \\
\begin{subarray} { c } { x / 2 < p _ { 1 } , \ldots , p _ { u } \leq x \\
( a b \Delta ( a , b ) , p _ { 1 } \cdots p _ { u } ) = 1 \\
p _ { r } \neq p _ { s } \text { if } 1 \leq r < s \leq u } }\end{subarray}} \prod_{j=1}^{u} \prod_{i \in \mathcal{S}_{j}} \tilde{a}_{E(a, b)}\left(p_{j}^{m_{i}}\right),
\end{aligned}
$$

where the second sum on the right-hand side runs over all partitions of the set $\{1, \ldots, t\}$ into $u$ disjoint non-empty sets $\mathcal{S}_{1}, \ldots, \mathcal{S}_{u}$.

10.2. Applying Lemma 3.1, Using Lemma 3.1, we have

$$
\prod_{i \in \mathcal{S}_{j}} \tilde{a}_{E(a, b)}\left(p_{j}^{m_{i}}\right)=\sum_{m=0}^{\infty} D\left(\left(m_{i}\right)_{i \in \mathcal{S}_{j}} ; m\right) \tilde{a}_{E(a, b)}\left(p_{j}^{m}\right)
$$

for all $j \in\{1, \ldots, u\}$ if $\left(\Delta(a, b), p_{1} \cdots p_{u}\right)=1$. From this and Theorem 2.1(iii), we further deduce that

$$
\prod_{j=1}^{u} \prod_{i \in \mathcal{S}_{j}} a_{E(a, b)}\left(p_{j}^{m_{i}}\right)=\sum_{\alpha_{1}=0}^{\infty} \cdots \sum_{\alpha_{u}=0}^{\infty}\left(\prod_{j=1}^{u} D\left(\left(m_{i}\right)_{i \in \mathcal{S}_{j}} ; \alpha_{j}\right)\right) \tilde{a}_{E(a, b)}\left(p_{1}^{\alpha_{1}} \cdots p_{u}^{\alpha_{u}}\right)
$$

under this condition. Combining (68), (69) and (70), and rearranging summations, we obtain

$$
\begin{aligned}
& X_{t}=\sum_{u=1}^{t} \sum_{\alpha_{1}=0}^{\infty} \cdots \sum_{\alpha_{u}=0}^{\infty} C\left(\alpha_{1}, \ldots, \alpha_{u}\right) \sum_{\substack{x / 2<p_{1}, \ldots, p_{u} \leq x \\
p_{r} \neq p_{s} \text { if } 1 \leq r<s \leq u}} \frac{1}{4 A B} \times \\
& \sum_{|a| \leq A} \sum_{|b| \leq B} \tilde{a}_{E(a, b)}\left(p_{1}^{\alpha_{1}} \cdots p_{u}^{\alpha_{u}}\right), \\
& \left(a b \Delta(a, b), p_{1} \cdots p_{u}\right)=1
\end{aligned}
$$


where

$$
C\left(\alpha_{1}, \ldots, \alpha_{u}\right):=\sum_{\{1, \ldots, t\}=\mathcal{S}_{1} \dot{\cup} \ldots \cup \mathcal{S}_{u}} \sum_{1 \leq m_{1}, \ldots, m_{t} \leq M} U\left(m_{1}\right) \cdots U\left(m_{t}\right) \prod_{j=1}^{u} D\left(\left(m_{i}\right)_{i \in \mathcal{S}_{j}} ; \alpha_{j}\right) .
$$

10.3. Estimation of $C\left(\alpha_{1}, \ldots, \alpha_{t}\right)$. Let

$$
z:=\sharp\left\{i \in\{1, \ldots, u\}: \alpha_{i}=0\right\} \quad \text { and } \quad n:=\sharp\left\{i \in\{1, \ldots, u\}: \alpha_{i} \neq 0\right\} .
$$

Then from Lemma 3.1 and $U\left(m_{i}\right) \ll 1 / m_{i}$, we deduce that

$$
\begin{aligned}
C\left(\alpha_{1}, \ldots, \alpha_{u}\right) & =O_{t}\left(\frac{M^{t-2 z-n}(\log M)^{t-u}}{\left(\alpha_{1}+1\right) \cdots\left(\alpha_{u}+1\right)}\right) \quad \text { if } 2 z+n \leq t \\
C\left(\alpha_{1}, \ldots, \alpha_{u}\right) & =0 \quad \text { if } 2 z+n>t \\
C(0, \ldots, 0) & =O_{t}\left(M^{t-2 z-1}(\log M)^{t-z}\right) \quad \text { if } 2 z<t \\
C(0, \ldots, 0) & =\frac{(2 z) !}{2^{z} z !} \cdot Z^{z} \quad \text { if } 2 z=t \\
C\left(\alpha_{1}, \ldots, \alpha_{u}\right) & =0 \quad \text { if } \alpha_{i}>t M \text { for an } i \in\{1, \ldots, u\},
\end{aligned}
$$

where $Z$ is defined as in (18). Here we use the first two equations in (27) if $\alpha_{i} \neq 0$ and the last three equations in (27) if $\alpha_{i}=0$.

10.4. Averaging over $a$ and $b$. Using Lemma 4.2 and Theorem 5.1 we have

$$
\begin{aligned}
& \frac{1}{4 A B} \sum_{\substack{|a| \leq A|b| \leq B \\
\left(a b \Delta(a, b), p_{1} \cdots p_{u}\right)=1}} \tilde{a}_{E(a, b)}\left(p_{1}^{\alpha_{1}} \cdots p_{u}^{\alpha_{u}}\right) \\
= & S\left(p_{1}^{\alpha_{1}}\right) \cdots S\left(p_{u}^{\alpha_{u}}\right)+O_{u}\left(\prod_{i=1}^{u}\left(\alpha_{i}+1\right) \cdot x^{u / 2+\varepsilon}\left(\frac{1}{A}+\frac{1}{B}\right)\right) .
\end{aligned}
$$

Combining this with (71), and using (73), (74), (75) and (78), we obtain

$$
\begin{aligned}
X_{t}= & \sum_{u=1}^{t} \sum_{\alpha_{1}=0}^{t M} \cdots \sum_{\alpha_{u}=0}^{t M} C\left(\alpha_{1}, \ldots, \alpha_{u}\right) \sum_{\substack{x / 2<p_{1}, \ldots, p_{u} \leq x \\
p_{r} \neq p_{s} \text { if } 1 \leq r<s \leq u}} S\left(p_{1}^{\alpha_{1}}\right) \cdots S\left(p_{u}^{\alpha_{u}}\right)+ \\
& O_{t}\left(M^{t+\varepsilon} x^{3 t / 2+\varepsilon}\left(\frac{1}{A}+\frac{1}{B}\right)\right) .
\end{aligned}
$$

10.5. Separating the primes. Next, we remove the summation conditon $p_{r} \neq p_{s}$ which was introduced to make use of the multiplicativity of the functions $\tilde{a}_{E(a, b)}(n)$ and $S(n)$. In this way, we make the prime variables $p_{j}$ independent.

We first observe that

$$
\begin{aligned}
& \sum_{\substack{x / 2<p_{1}, \ldots, p_{u} \leq x \\
p_{r} \neq p_{s} \text { if } 1 \leq r<s \leq u}} S\left(p_{1}^{\alpha_{1}}\right) \cdots S\left(p_{u}^{\alpha_{u}}\right)=(\tilde{\pi}(x)-n) \cdots(\tilde{\pi}(x)-u+1) \times \\
& \sum_{\substack{x / 2<p_{1}, \ldots, p_{n} \leq x \\
p_{r} \neq p_{s} \text { if } 1 \leq r<s \leq n}} S\left(p_{1}^{\beta_{1}}\right) \cdots S\left(p_{n}^{\beta_{n}}\right),
\end{aligned}
$$

where $z$ and $n$ are defined as in (73), and $\left(\beta_{1}, \ldots, \beta_{n}\right)$ is the $n$-tuple obtained by removing all zero elements from the $u$-tuple $\left(\alpha_{1}, \ldots, \alpha_{u}\right)$. To see (80), we note that if $n$ distinct primes $p_{1}, \ldots, p_{n}$ in $(x / 2, x]$ are fixed, then there are $\tilde{\pi}(x)-n$ possibilities to choose a prime $p_{n+1}$ in $(x / 2, x]$ distinct from $p_{1}, \ldots, p_{n}, \tilde{\pi}(x)-n-1$ possibilities to choose another prime $p_{n+2}$ in $(x / 2, x]$ distinct from $p_{1}, \ldots, p_{n+1}$, and so on. We further note that

$$
(\tilde{\pi}(x)-n) \cdots(\tilde{\pi}(x)-u+1)=\tilde{\pi}(x)^{z}+O\left(\tilde{\pi}(x)^{z-1}\right) .
$$


Now we claim that the right-hand side of (80) can be written as a sum over partitions $\mathcal{P}$ of the set $\{1, \ldots, n\}$ in the form

$$
\sum_{\substack{x / 2<p_{1}, \ldots, p_{n} \leq x \\ p_{r} \neq p_{s} \text { if } 1 \leq r<s \leq n}} S\left(p_{1}^{\beta_{1}}\right) \cdots S\left(p_{n}^{\beta_{n}}\right)=\sum_{\substack{\mathcal{P}=\left\{\mathcal{M}_{1}, \ldots, \mathcal{M}_{k}\right\} \\ \mathcal{M}_{1} \cup \cdots \cup \mathcal{M}_{k}=\{1, \ldots, n\}}} A(\mathcal{P}) \cdot \prod_{l=1}^{k} \sum_{x / 2<p \leq x} \prod_{j \in \mathcal{M}_{l}} S\left(p^{\beta_{j}}\right),
$$

where

$$
A(\mathcal{P})=A\left(\left\{\mathcal{M}_{1}, \ldots, \mathcal{M}_{k}\right\}\right)=(-1)^{n-k} \cdot \prod_{l=1}^{k}\left(\sharp \mathcal{M}_{l}-1\right) ! .
$$

To establish (82), we use the identity

$$
\begin{aligned}
& \sum_{\substack{x / 2<p_{1}, \ldots, p_{n} \leq x \\
p_{r} \neq p_{s} \text { if } 1 \leq r<s \leq n}} S\left(p_{1}^{\beta_{1}}\right) \cdots S\left(p_{n}^{\beta_{n}}\right)=\sum_{\substack{x / 2<p_{1}, \ldots, p_{n} \leq x \\
p_{r} \neq p_{s} \text { if } 1 \leq r<s \leq n-1}} S\left(p_{1}^{\beta_{1}}\right) \cdots S\left(p_{n}^{\beta_{n}}\right)- \\
& \sum_{w=1}^{n-1} \sum_{\substack{x / 2<p_{1}, \ldots, p_{n} \leq x \\
p_{r} \neq p_{s} \text { if } 1 \leq r<\leq \leq n-1 \\
p_{n}=p_{w} \leq n-1}} S\left(p_{1}^{\beta_{1}}\right) \cdots S\left(p_{n}^{\beta_{n}}\right)
\end{aligned}
$$

for $n \geq 2$ and proceed by induction over $n$. In this way, we see that

$$
\begin{aligned}
& \sum_{\substack{x / 2<p_{1}, \ldots, p_{n} \leq x \\
p_{r} \neq p_{s} \text { if } 1 \leq r<s \leq n}} S\left(p_{1}^{\beta_{1}}\right) \cdots S\left(p_{n}^{\beta_{n}}\right) \\
& =\sum_{\substack{\mathcal{P}^{\prime}=\left\{\mathcal{M}_{1}, \ldots, \mathcal{M}_{k}\right\} \\
\mathcal{M}_{1} \cup \ldots \cup \mathcal{M}_{k}=\{1, \ldots, n-1\}}} A\left(\mathcal{P}^{\prime}\right) \cdot\left(\prod_{l=1}^{k} \sum_{x / 2<p \leq x} \prod_{j \in \mathcal{M}_{l}} S\left(p^{\beta_{j}}\right)\right) \cdot\left(\sum_{x / 2<p \leq x} S\left(p^{\beta_{n}}\right)\right)- \\
& \sum_{\substack{\mathcal{P}^{\prime}=\left\{\mathcal{M}_{1}, \ldots, \mathcal{M}_{k}\right\} \\
\mathcal{M}_{1} \cup \ldots \cup \mathcal{M}_{k}=\{1, \ldots, n-1\}}} A\left(\mathcal{P}^{\prime}\right) \cdot \sum_{m=1}^{k}\left(\sharp \mathcal{M}_{m}\right) \cdot\left(\prod_{\substack{l=1 \\
l \neq m}}^{k} \sum_{x / 2<p \leq x} \prod_{j \in \mathcal{M}_{l}} S\left(p^{\beta_{j}}\right)\right) \times \\
& \left(\sum_{x / 2<p \leq x} S\left(p^{\beta_{n}}\right) \cdot \prod_{j \in \mathcal{M}_{m}} S\left(p^{\beta_{j}}\right)\right) \\
& =\sum_{\substack{\mathcal{P}=\left\{\mathcal{M}_{1}, \ldots, \mathcal{M}_{k}\right\} \\
\mathcal{M}_{1} \cup \ldots \cup \mathcal{M}_{k}=\{1, \ldots, n\}}} A(\mathcal{P}) \cdot \prod_{l=1}^{k} \sum_{x / 2<p \leq x} \prod_{j \in \mathcal{M}_{l}} S\left(p^{\beta_{j}}\right),
\end{aligned}
$$

where the coefficients satisfy the recursive relations

$$
\begin{aligned}
A(\{\{1\}\}) & =1, \\
A\left(\mathcal{P}^{\prime} \dot{\cup}\{\{n\}\}\right) & =A\left(\mathcal{P}^{\prime}\right), \\
A\left(\left\{\mathcal{M}_{1}, \ldots, \mathcal{M}_{m-1}, \mathcal{M}_{m} \cup\{n\}, \mathcal{M}_{m+1}, \ldots, \mathcal{M}_{k}\right\}\right) & =-\left(\sharp \mathcal{M}_{m}\right) \cdot A\left(\mathcal{P}^{\prime}\right)
\end{aligned}
$$

if $\mathcal{P}^{\prime}=\left\{\mathcal{M}_{1}, \ldots, \mathcal{M}_{k}\right\}$ and $\mathcal{M}_{1} \dot{\cup} \ldots \dot{\cup} \mathcal{M}_{k}=\{1, \ldots, n-1\}$. These recursive relations imply the explicit formula (83) via another induction argument.

10.6. Estimation of $X_{t}$ under MRH. Throughout the following subsections, let $F, c, \varepsilon>0$ be arbitrary but fixed constants and $0 \leq \beta_{i} \leq t M$ for $i \in\{1, \ldots, n\}$.

We first estimate $X_{t}$ under MRH. Assume that

$$
\tilde{\pi}(x)^{1 / 2} \leq M \leq x^{F} \quad \text { and } \quad A, B \geq x^{3 t / 2+(F+2) \varepsilon} .
$$


Then using parts (ii) and (v) of Lemma 9.2, we deduce from (82) that, under MRH,

$$
\sum_{\substack{x / 2<p_{1}, \ldots, p_{n} \leq x \\ p_{r} \neq p_{s} \text { if } 1 \leq r<s \leq n}} S\left(p_{1}^{\beta_{1}}\right) \cdots S\left(p_{n}^{\beta_{n}}\right) \ll_{n, F} \beta_{1} \cdots \beta_{n}(\log x)^{n} .
$$

Combining (74), (75), (79), (80), (81), (85) and (86), we obtain

$$
X_{t}=O_{t, F, \varepsilon}\left(M^{t}(\log x)^{t}\right) \quad \text { under MRH. }
$$

10.7. Unconditonal estimation of $X_{t}$. Next, we estimate $X_{t}$ unconditionally in a similar way. Assume that

$$
x^{\varepsilon} \leq M \leq x^{F} \quad \text { and } \quad A, B \geq x^{t+(F+2) \varepsilon} .
$$

Then using parts (i) and (v) of Lemma 9.2, we deduce from (82) that

$$
\sum_{\substack{x / 2<p_{1}, \ldots, p_{n} \leq x \\ p_{r} \neq p_{s} \text { if } 1 \leq r<s \leq n}} S\left(p_{1}^{\beta_{1}}\right) \cdots S\left(p_{n}^{\beta_{n}}\right) \ll_{n, F, c} \beta_{1} \cdots \beta_{n} x^{n / 2}(\log x)^{-(t+c)} .
$$

Combining (74), (75), (79), (80), (81), (87) and (88), we obtain

$$
X_{t}=O_{t, F, c, \varepsilon}\left(M^{t} x^{t / 2}(\log x)^{-c}\right) \quad \text { unconditionally. }
$$

10.8. Estimation of $X_{t}$ under Hypotheses 1,2. Finally, we estimate $X_{t}$ under Hypotheses 1 and 2. Assume that

$$
\tilde{\pi}(x)^{1 / 2} \leq M \leq x^{F} \quad \text { and } \quad A, B \geq x^{3 t / 2+(F+2) \varepsilon} .
$$

Using (82) and the triangle inequality, we have

$$
\begin{aligned}
& \sum_{1 \leq \beta_{1}, \ldots, \beta_{n} \leq t M} \frac{1}{\beta_{1} \cdots \beta_{n}} \cdot\left|\sum_{\substack{x / 2<p_{1}, \ldots, p_{n} \leq x \\
p_{r} \neq p_{s} \text { if } 1 \leq r<s \leq n}} S\left(p_{1}^{\beta_{1}}\right) \cdots S\left(p_{n}^{\beta_{n}}\right)\right| \\
\leq & \sum_{\substack{\mathcal{P}=\left\{\mathcal{M}_{1}, \ldots, \mathcal{M}_{k}\right\} \\
\mathcal{M}_{1} \cup \ldots \cup \mathcal{M}_{k}=\{1, \ldots, n\}}}|A(\mathcal{P})| \cdot \prod_{l=1}^{k}\left|\sum_{\substack{x / 2<p \leq x \\
j \in \mathcal{M}_{l}}} \sum_{1 \leq \beta_{j} \leq t M} \frac{S\left(p^{\beta_{j}}\right)}{\beta_{j}}\right| .
\end{aligned}
$$

From Lemma 9.2 and (90), we deduce that

$$
\sum_{1 \leq \beta_{1}, \ldots, \beta_{n} \leq t M} \frac{1}{\beta_{1} \cdots \beta_{n}} \cdot\left|\sum_{\substack{x / 2<p_{1}, \ldots, p_{n} \leq x \\ p_{r} \neq p_{s} \text { if } 1 \leq r<s \leq n}} S\left(p_{1}^{\beta_{1}}\right) \cdots S\left(p_{n}^{\beta_{n}}\right)\right| \ll_{n, F, c, t} M^{n}(\log x)^{-(t+c)},
$$

where we use parts (iii) and (v) of Lemma 9.2 if $n>2$, parts (iii) and (iv) if $n=2$, and part (iii) if $n=1$. Combining (74), (75), (76), (77), (79), (80), (81), (89), and (91), we obtain

$$
X_{t}=\delta(t) \cdot \frac{t !}{2^{t / 2}(t / 2) !} \cdot(Z \tilde{\pi}(x))^{t / 2}+O_{t, F, c, \varepsilon}\left(M^{t}(\log x)^{-c}\right) \quad \text { under Hypotheses } 1 \text { and } 2 .
$$

Combining the results of the last three subsections, we obtain claimed estimate (19) upon changing the term $(F+2) \varepsilon$ in the conditions on $A$ and $B$ into $\varepsilon$. This completes the proof. 


\section{Proof of Theorem 1.11}

We recall that the summation condition $p \nmid a b$, which was present on the left-hand side of (19), is omitted in (20). To prove Theorem 1.11, we proceed in the same way as in the proof of Theorem 1.10, where we replace the estimate (35) by

$$
\sum_{\substack{|a| \leq A \\(\Delta(a, b), n)=1}} \tilde{a}_{E(a, b)}(n)=4 A B S_{0}(n)+O\left(d(n) s(n)^{2}\right)+O(d(n) s(n)(A+B)),
$$

which can be established in a similar way as (31). We further use the fact that $S_{0}(n)$ is multiplicative just like $S(n)$ is multiplicative by Lemma 4.2 and employ Lemma 9.3, which holds under Hypothesis 1 only, instead of Lemma 9.2(iii). Avoiding Hypothesis 2 comes at the cost of replacing the condition $A, B \geq x^{3 t / 2+\varepsilon}$ in the third estimate on the right-hand side of (19) by the stronger condition $A, B \geq x^{2 t+\varepsilon}$, which is due to the weaker $O$-term in (92) in place of the $O$-term in (35).

\section{Proof of Theorem 1.4}

Let $M \in \mathbb{N}$, to be fixed later.

12.1. Removing the primes $p$ dividing $a b$. We start our proof with getting rid of the contribution of primes $p$ dividing $a b$. First, we separate the contribution of $a b=0$, observing that

$$
\begin{aligned}
& \frac{1}{4 A B} \sum_{|a| \leq A} \sum_{|b| \leq B}\left(N_{I}(E(a, b), x)-\tilde{\pi}(x) \mu(I)\right)^{t} \\
= & \frac{1}{4 A B} \sum_{0<|a| \leq A} \sum_{0<|b| \leq B}\left(N_{I}(E(a, b), x)-\tilde{\pi}(x) \mu(I)\right)^{t}+O\left(x^{t}\left(\frac{1}{A}+\frac{1}{B}\right)\right)
\end{aligned}
$$

by a trivial estimation. Now it suffices to treat the case $a b \neq 0$. We recall the definitions of $U_{I}^{ \pm}(m)$ and $P_{I}^{ \pm}(E, x)$ in Theorem 1.8 and deduce the bound

$$
\sum_{1 \leq m \leq M} U_{I}^{ \pm}(m) \tilde{a}_{E}\left(p^{m}\right)=O(\log 2 M)
$$

for every prime $p$ of good reduction at $E$ from the bound

$$
\tilde{a}_{E}\left(p^{m+2}\right)-\tilde{a}_{E}\left(p^{m}\right)=O(1),
$$

which follows from the fact that $\tilde{a}_{E}\left(p^{m+2}\right)-\tilde{a}_{E}\left(p^{m}\right)=2 \cos \left(m \theta_{E}(p)\right)$ where $\tilde{a}_{E}(p)=2 \cos \left(\theta_{E}(p)\right)$. For details, see Corollary 2.3 of [22]. From (16), (94) and the well-known bound $\omega(a b) \ll \log (2|a b|)$ for the number of prime divisors of $a b$, we deduce that

$$
\begin{aligned}
& \tilde{P}_{I}^{-}(E(a, b), x)+O\left(\frac{\tilde{\pi}(x)}{M}+\log (2|a b|) \log (2 M)\right) \\
\leq & N_{I}(E(a, b), x)-\tilde{\pi}(x) \mu(I) \leq \tilde{P}_{I}^{+}(E(a, b), x)+O\left(\frac{\tilde{\pi}(x)}{M}+\log (2|a b|) \log (2 M)\right)
\end{aligned}
$$

if $a b \neq 0$, where

$$
\begin{gathered}
\tilde{P}_{I}^{ \pm}(E(a, b), x):=\sum_{1 \leq m \leq M} U_{I}^{ \pm}(m) \sum_{\substack{x / 2<p \leq x \\
p \nmid a b \Delta(a, b)}} \tilde{a}_{E(a, b)}\left(p^{m}\right) \\
=P_{I}^{ \pm}(E(a, b), x)-\sum_{1 \leq m \leq M} U_{I}^{ \pm}(m) \sum_{\substack{x / 2<p \leq x \\
p \nmid \Delta(a, b) \\
p \mid a b}} \tilde{a}_{E(a, b)}\left(p^{m}\right) .
\end{gathered}
$$


12.2. Application of the binomial formula. Next, we use the binomial formula to write

$$
\left(N_{I}(E, x)-\tilde{\pi}(x) \mu(I)\right)^{t}=\sum_{s=0}^{t}\left(\begin{array}{l}
t \\
s
\end{array}\right)\left(N_{I}(E, x)-\tilde{\pi}(x) \mu(I)-\tilde{P}_{I}^{-}(E, x)\right)^{t-s} \tilde{P}_{I}^{-}(E, x)^{s} .
$$

Using the Cauchy-Schwarz inequality and taking into acoount that $p \mid a b \Delta(a, b)$ for every prime $p$ if $a=0$ or $b=0$, we deduce that

$$
\begin{aligned}
& \frac{1}{4 A B} \sum_{0<|a| \leq A} \sum_{0<|b| \leq B}\left(N_{I}(E(a, b), x)-\tilde{\pi}(x) \mu(I)\right)^{t} \\
= & \frac{1}{4 A B} \sum_{|a| \leq A} \sum_{|b| \leq B} \tilde{P}_{I}^{-}(E(a, b), x)^{t}+ \\
& O_{t}\left(\frac{1}{4 A B} \sum_{|a| \leq A} \sum_{|b| \leq B}\left(N_{I}(E(a, b), x)-\tilde{\pi}(x) \mu(I)-\tilde{P}_{I}^{-}(E(a, b), x)\right)^{t}+\right. \\
& \sum_{s=1}^{t-1}\left(\frac{1}{4 A B} \sum_{|a| \leq A|b| \leq B} \sum_{I} \tilde{P}_{I}^{-}(E(a, b), x)^{2 s}\right)^{1 / 2} \times \\
& \left.\left(\frac{1}{4 A B} \sum_{|a| \leq A|b| \leq B} \sum\left(N_{I}(E(a, b), x)-\tilde{\pi}(x) \mu(I)-\tilde{P}_{I}^{-}(E(a, b), x)\right)^{2(t-s)}\right)^{1 / 2}\right) .
\end{aligned}
$$

12.3. Estimation of moments. We need to evaluate the $v$-th moment of $P_{I}^{-}(E(a, b), x)$ for $v=2 s$ and $v=t$. Applying Theorems 1.9 and 1.10, we get

$$
\begin{aligned}
& \frac{1}{4 A B} \sum_{|a| \leq A} \sum_{|b| \leq B} \tilde{P}_{I}^{-}(E(a, b), x)^{v} \\
= & \delta(v) \cdot \frac{v !}{2^{v / 2}(v / 2) !} \cdot\left(\mu(I)-\mu(I)^{2}+\frac{\log (2 M)}{M}\right)^{v / 2}\left(\tilde{\pi}(x)^{v / 2}+O\left(\tilde{\pi}(x)^{v / 2-1}\right)\right)+ \\
& \begin{cases}O_{v, F, c, \varepsilon}\left(M^{v} x^{v / 2}(\log x)^{-c}\right) & \text { unconditionally if } x^{\varepsilon} \leq M \leq x^{F} \text { and } A, B \geq x^{v+\varepsilon} \\
O_{v, F, \varepsilon}\left(M^{v}(\log x)^{v}\right) & \text { under MRH if } \tilde{\pi}(x)^{1 / 2} \leq M \leq x^{F} \text { and } A, B \geq x^{3 v / 2+\varepsilon} \\
O_{v, F, c, \varepsilon}\left(M^{v}(\log x)^{-c}\right) & \text { under Hyp.1,2 if } \tilde{\pi}(x)^{1 / 2} \leq M \leq x^{F} \text { and } A, B \geq x^{3 v / 2+\varepsilon} .\end{cases}
\end{aligned}
$$

We further need to evaluate the $v$-th moments of $N_{I}(E(a, b), x)-\tilde{\pi}(x) \mu(I)-\tilde{P}_{I}^{-}(E(a, b), x)$ for $v=2(t-s)$, which is even, and $v=t$, which is possibly odd. Using (95), we observe that

$$
\begin{aligned}
-\frac{K \tilde{\pi}(x)}{M} & \leq N_{I}(E(a, b), x)-\tilde{\pi}(x) \mu(I)-\tilde{P}_{I}^{-}(E(a, b), x) \\
& \leq \frac{L \tilde{\pi}(x)}{M}+\tilde{P}_{I}^{+}(E(a, b), x)-P_{I}^{-}(E(a, b), x)
\end{aligned}
$$

for some absolute constants $K, L>0$, provided that

$$
\log (2|a b|) \log (2 M) \leq \log (2 A B) \log (2 M) \leq \frac{\tilde{\pi}(x)}{M},
$$

which we want to assume from now on. We further note that

$$
\tilde{P}_{I}^{+}(E(a, b), x)-\tilde{P}_{I}^{-}(E(a, b), x)=\sum_{1 \leq m \leq M}\left(U_{I}^{+}(m)-U_{I}^{-}(m)\right) \sum_{\substack{x / 2<p \leq x \\ p \nmid a b \Delta(a, b)}} \tilde{a}_{E(a, b)}\left(p^{m}\right)
$$

and

$$
U_{I}^{+}(m)-U_{I}^{-}(m) \ll \frac{1}{M} \text { for } 1 \leq m \leq M
$$


If $v$ is even, then, using the above considerations and the inequality

$$
\left(N_{I}(E(a, b), x)-\tilde{\pi}(x) \mu(I)-\tilde{P}_{I}^{-}(E(a, b), x)\right)^{v} \ll_{v}\left(\frac{\tilde{\pi}(x)}{M}\right)^{v}+\left(\tilde{P}_{I}^{+}(E(a, b), x)-\tilde{P}_{I}^{-}(E(a, b), x)\right)^{v}
$$

following from (98), we deduce from Theorem 1.10 with $F=1$ that

$$
\begin{aligned}
& \frac{1}{4 A B} \sum_{|a| \leq A|b| \leq B}\left(N_{I}(E(a, b), x)-\tilde{\pi}(x) \mu(I)-\tilde{P}_{I}^{-}(E(a, b), x)\right)^{v} \\
= & O_{v}\left(\frac{\tilde{\pi}(x)^{v / 2} \log (2 M)}{M}+\left(\frac{\tilde{\pi}(x)}{M}\right)^{v}\right)+ \\
& \begin{cases}O_{v, c, \varepsilon}\left(M^{v} x^{v / 2}(\log x)^{-c}\right) & \text { unconditionally if } x^{\varepsilon} \leq M \leq \tilde{\pi}(x) \text { and } A, B \geq x^{v+\varepsilon} \\
O_{v, \varepsilon}\left(M^{v}(\log x)^{v}\right) & \text { under MRH if } \tilde{\pi}(x)^{1 / 2} \leq M \leq \tilde{\pi}(x) \text { and } A, B \geq x^{3 v / 2+\varepsilon} \\
O_{v, c, \varepsilon}\left(M^{v}(\log x)^{-c}\right) & \text { under Hyp.1,2 if } \tilde{\pi}(x)^{1 / 2} \leq M \leq \tilde{\pi}(x) \text { and } A, B \geq x^{3 v / 2+\varepsilon} .\end{cases}
\end{aligned}
$$

If $v$ is odd, then we need to argue more carefully. Here we use the fact that

$$
\begin{aligned}
-\left(\frac{K \tilde{\pi}(x)}{M}\right)^{v} & \leq\left(N_{I}(E(a, b), x)-\tilde{\pi}(x) \mu(I)-\tilde{P}_{I}^{-}(E(a, b), x)\right)^{v} \\
& \leq\left(\frac{L \tilde{\pi}(x)}{M}+\tilde{P}_{I}^{+}(E(a, b), x)-\tilde{P}_{I}^{-}(E(a, b), x)\right)^{v} \\
& =\sum_{s=0}^{v}\left(\begin{array}{l}
v \\
s
\end{array}\right)\left(\frac{L \tilde{\pi}(x)}{M}\right)^{v-s}\left(\tilde{P}_{I}^{+}(E(a, b), x)-\tilde{P}_{I}^{-}(E(a, b), x)\right)^{s},
\end{aligned}
$$

which follows from (98) as well. Applying Theorem 1.10 again, we see after a short calculation that the same bound as in (100) holds in this case too.

\subsection{Completion of the proof. Now we choose}

$$
M:= \begin{cases}\left\lceil x^{1 / 4}(\log x)^{c /(2 t)}\right\rceil & \text { unconditionally } \\ \left\lceil\tilde{\pi}(x)^{1 / 2}\right\rceil & \text { under MRH } \\ \left\lceil x^{1 / 2}(\log x)^{c /(2 t)}\right\rceil & \text { under Hypotheses 1,2 }\end{cases}
$$

and impose the condition that

$$
A B \leq \exp \left(x^{1 / 2-\varepsilon}\right)
$$

so that (99) is satisfied in each case if $x$ is large enough. Then, if $1 \leq v \leq t$, (97) simplifies into

$$
\begin{aligned}
& \frac{1}{4 A B} \sum_{|a| \leq A} \sum_{|b| \leq B} \tilde{P}_{I}^{-}(E(a, b), x)^{v}=\delta(v) \cdot \frac{v !}{2^{v / 2}(v / 2) !} \cdot \tilde{\pi}(x)^{v / 2}\left(\mu(I)-\mu(I)^{2}\right)^{v / 2}+ \\
& \begin{cases}O_{v, c, \varepsilon}\left(x^{3 v / 4}(\log x)^{-c / 2}\right) & \text { unconditionally if } A, B \geq x^{v+\varepsilon} \\
O_{v, \varepsilon}\left(\tilde{\pi}(x)^{v / 2}(\log x)^{v}\right) & \text { under MRH if } A, B \geq x^{3 v / 2+\varepsilon} \\
O_{v, c, \varepsilon}\left(x^{v / 2}(\log x)^{-c / 2}\right) & \text { under Hyp.1,2 if } A, B \geq x^{3 v / 2+\varepsilon},\end{cases}
\end{aligned}
$$

and (100) simplifies into

$$
\begin{aligned}
& \frac{1}{4 A B} \sum_{|a| \leq A} \sum_{|b| \leq B}\left(N_{I}(E(a, b), x)-\tilde{\pi}(x) \mu(I)-\tilde{P}_{I}^{-}(E(a, b), x)\right)^{v} \\
= & \begin{cases}O_{v, c, \varepsilon}\left(x^{3 v / 4}(\log x)^{-c / 2}\right) & \text { unconditionally if } A, B \geq x^{v+\varepsilon} \\
O_{v, \varepsilon}\left(\tilde{\pi}(x)^{v / 2}(\log x)^{v}\right) & \text { under MRH if } A, B \geq x^{3 v / 2+\varepsilon} \\
O_{v, c, \varepsilon}\left(x^{v / 2}(\log x)^{-c / 2}\right) & \text { under Hyp.1,2 if } A, B \geq x^{3 v / 2+\varepsilon} .\end{cases}
\end{aligned}
$$

Combining (96), (102) and (103) gives the first three estimates in (12) upon changing $c$ into $2 c$ in the unconditional case and into $4 t c$ under Hypotheses 1, 2. In our computations, we take into account that the main term on the right hand side of (102) may dominate under Hypotheses 
1,2. The fourth estimate is established in a similar way, but here we avoid removing the primes $p$ dividing $a b$, as carried out in subsection 12.1. work directly with the polynomials $P_{I}^{ \pm}(E(a, b), x)$ instead of $\tilde{P}_{I}^{ \pm}(E(a, b), x)$, and apply Theorem 1.11 in place of Theorem 1.10, where the truth of Hypothesis 2 is not assumed. We note that avoiding the treatment in subsection 12.1 also saves us from assuming that $A B \leq \exp \left(x^{1 / 2-\varepsilon}\right)$. Finally, we point out that we need to introduce the function $\eta(t)$ in the conditions on $A$ and $B$ in (12) because of the use of the Cauchy-Schwarz inequality in (96). The latter introduces the powers $2 s$ and $2(t-s)$ which go up to $2(t-1)$. Now the proof of Theorem 1.4 is complete.

\section{REFERENCES}

[1] Akiyama, S.; Tanigawa, Y., Calculation of elliptic L functions in the critical strip, Sũrikaisekikenkyũsho Kõkyũroku No. 961 (1996) 11-31.

[2] Baier, S., The Lang-Trotter conjecture on average, J. Ramanujan Math. Soc. 22 (2007) 299-314.

[3] Baier, S., A remark on the Lang-Trotter conjecture, New directions in value-distribution theory of zeta and L-functions, 1118, Ber. Math., Shaker Verlag, Aachen, 2009.

[4] Baier, S.; Zhao, L., The Sato-Tate conjecture on average for small angles, Trans. Amer. Math. Soc. 361 (2009) 1811-1832.

[5] Banks, W.D.; Shparlinski, I.E., Sato-Tate, cyclicity, and divisibility statistics on average for elliptic curves of small height, Israel J. Math., 173 (2009) 253-277.

[6] Birch, B.J., How the number of points of an elliptic curve over a fixed prime field varies, J. London Math. Soc. 43 (1968) 57-60.

[7] Barnet-Lamb, T.; Geraghty. D.; Harris, M.; Taylor, R., A family if Calabi Yau varieties and potential automorphy II, Publ. Res. Inst. Math. Sci. 47 (2011) 29-98.

[8] Clozel, L.; Harris, M,; Taylor, R., Automorphy for some l-adic lifts of automorphic mod l Galois representations, Publ. Math. Inst. Hautes tudes Sci. 108 (2008) 1-181.

[9] David, C.; Koukoulopulos, D.; Smith, E., Sums of Euler products and statistics of elliptic curves, to appear in Math. Ann., arXiv:1510.05935

[10] Deligne, P., La conjecture de Weil. I., Inst. Hautes tudes Sci. Publ. Math. No. 43 (1974) 273-307.

[11] Deuring, M., Die Typen der Multiplikatorenringe elliptischer Funktionenkörper, Abh. Math. Sem. Hansischen Univ. 14 (1941) 197-272.

[12] Harris, M.; Shepherd-Barron, N.; Taylor, R. A family of CalabiYau varieties and potential automorphy, Ann. Math., 171 (2010) 779-813.

[13] Iwaniec, H.; Kowalski, E., Analytic number theory, American Mathematical Society Colloquium Publications 53, American Mathematical Society, Providence, RI, 2004.

[14] Lang, S., Introduction to modular forms. With appendixes by D. Zagier and Walter Feit, Corrected reprint of the 1976 original. Grundlehren der Mathematischen Wissenschaften [Fundamental Principles of Mathematical Sciences], 222. Springer-Verlag, Berlin, 1995. x+261 pp.

[15] Langlands, R.P., Problems in the theory of automorphic forms, Lectures in modern analysis and applications, III, pp. 1861. Lecture Notes in Math., Vol. 170, Springer, Berlin, 1970.

[16] Mazur, B., Finding meaning in error terms, Bull. Amer. Math. Soc. 45 (2008) 185-228.

[17] Melzak, Z.A., Problem 4458, Amer. Math. Monthly 58 (1951) 636.

[18] Murty, M.R., Applications of symmetric power L-functions, http://www.mast.queensu.ca/ murty/Murty.pdf

[19] Murty, V.K., Explicit formulae and the Lang-Trotter conjecture, Rocky Mountain J. Math 15 (1985) 535-551.

[20] Prabhu, N., Fluctuations in the distribution of Hecke eigenvalues, PhD Thesis, Indian Institute of Science Education and Reasearch, Pune, India.

[21] M. Sha, I. Shparlinski, Lang-Trotter and Sato-Tate distributions in single and double parametric families of elliptic curves, Acta Arith. 170 (2015) 299-325.

[22] Prabhu, N.; Sinha, K., Fluctuations in the distribution of Hecke eigenvalues about the Sato-Tate measure, Int. Math. Res. Not., https://doi.org/10.1093/imrn/rnx238

[23] Silverman, J.H., The arithmetic of elliptic curves, Second edition. Graduate Texts in Mathematics, 106. Springer, Dordrecht, 2009. xx+513 pp.

[24] Taylor, R., Automorphy for some l-adic lifts of automorphic mod l Galois representations II, Publ. Math. Inst. Hautes tudes Sci. 108 (2008) 183-239.

Stephan Baier, Ramakrishna Mission Vivekananda University, Department of Mathematics, Po Belur

Math, Dist Howrah 711202, West Bengal, India

E-mail address, Stephan Baier: email_baier@yahoo.de

Neha Prabhu, Queen's University, Kingston, Ontario K7K 3N6

E-mail address, Neha Prabhu: neha.prabhu@queensu.ca 